\title{
Inside-out Planet Formation. IV. Pebble Evolution and Planet Formation Timescales
}

\author{
Xiao Hu (胡晓) $)^{1,2}$ (1) , Jonathan C. Tan ${ }^{1,3}$ (1) Z Zhaohuan Zhu (朱照寰) $)^{2}$ (1), Sourav Chatterjee ${ }^{4}$ (1) , Tilman Birnstiel ${ }^{5}$ (1), \\ Andrew N. Youdin ${ }^{6}$, and Subhanjoy Mohanty ${ }^{7}$ (D) \\ ${ }^{1}$ Department of Astronomy, University of Florida, Gainesville, FL 32611, USA \\ ${ }^{2}$ Department of Physics and Astronomy, University of Nevada, Las Vegas, 4505 South Maryland Parkway, Las Vegas, NV 89154, USA \\ ${ }^{3}$ Department of Physics, University of Florida, Gainesville, FL 32611, USA \\ ${ }^{4}$ Center for Interdisciplinary Exploration and Research in Astrophysics (CIERA), Physics and Astronomy, Northwestern University, Evanston, IL 60208, USA \\ ${ }^{5}$ University Observatory, Faculty of Physics, Ludwig-Maximilians-Universität München, Scheinerstr. 1, D-81679 Munich, Germany \\ ${ }^{6}$ Department of Astronomy and Steward Observatory, University of Arizona, 933 North Cherry Avenue, Tucson, AZ 85721, USA \\ ${ }^{7}$ Imperial College London, 1010 Blackett Lab, Prince Consort Rd., London SW7 2AZ, UK \\ Received 2017 September 28; revised 2018 January 18; accepted 2018 February 1; published 2018 April 9
}

\begin{abstract}
Systems with tightly packed inner planets (STIPs) are very common. Chatterjee \& Tan proposed Inside-out Planet Formation (IOPF), an in situ formation theory, to explain these planets. IOPF involves sequential planet formation from pebble-rich rings that are fed from the outer disk and trapped at the pressure maximum associated with the dead zone inner boundary (DZIB). Planet masses are set by their ability to open a gap and cause the DZIB to retreat outwards. We present models for the disk density and temperature structures that are relevant to the conditions of IOPF. For a wide range of DZIB conditions, we evaluate the gap-opening masses of planets in these disks that are expected to lead to the truncation of pebble accretion onto the forming planet. We then consider the evolution of dust and pebbles in the disk, estimating that pebbles typically grow to sizes of a few centimeters during their radial drift from several tens of astronomical units to the inner, $\lesssim 1$ au scale disk. A large fraction of the accretion flux of solids is expected to be in such pebbles. This allows us to estimate the timescales for individual planet formation and the entire planetary system formation in the IOPF scenario. We find that to produce realistic STIPs within reasonable timescales similar to disk lifetimes requires disk accretion rates of $\sim 10^{-9} M_{\odot} \mathrm{yr}^{-1}$ and relatively low viscosity conditions in the DZIB region, i.e., a Shakura-Sunyaev parameter of $\alpha \sim 10^{-4}$.
\end{abstract}

Key words: accretion, accretion disks - planet-disk interactions - planetary systems - planets and satellites: formation - protoplanetary disks

\section{Introduction}

More than 4000 exoplanet candidates (e.g., Mullally et al. 2015) have been discovered by Kepler since 2009, a significant percentage $(\gtrsim 30 \%)$ of which are in systems with tightly packed inner planets (hereafter STIPs). STIPs are very different from our solar system: they typically contain three or more detected planets of radii $\sim 1-10 R_{\oplus}$ and with periods less than 100 days (Fang \& Margot 2012), i.e., with orbital radii of $\lesssim 0.3$ au.

One way to form STIPs may be via the inward migration of planets formed in the outer disk (e.g., Kley \& Nelson 2012; Cossou et al. 2013, 2014). However, it can be quite difficult to concentrate planets close to the host star to the degree observed in STIPs (McNeil \& Nelson 2010). Also, migrating planets tend to become trapped in orbits of low-order mean motion resonances, which is not a particular feature of STIPs (Baruteau et al. 2014; Fabrycky et al. 2014). This lack of resonant pile-up has motivated studies of mechanisms that cause a lower efficiency of resonance trapping (Goldreich \& Schlichting 2014) or the breaking of resonance by later dynamical interactions with, e.g., a planetesimal disk (Chatterjee \& Ford 2015), by the unstable nature of compact resonant chains (Izidoro et al. 2017), or by magnetospheric rebound from the host star during gas disk dispersal (Liu et al. 2017).

As an alternative to inward migration, in situ formation scenarios (Hansen \& Murray 2012, 2013) have considered a group of protoplanets that are initially distributed inside 1 au with a high concentration. After following the dynamical evolution for $10 \mathrm{Myr}$, collisional oligarchic growth can lead to systems quite similar to STIPs. However, how this initial condition was established was not well-studied in these works and may be subject to some difficulties, such as the early triggering of gravitational instability (Raymond \& Cossou 2014; Schlichting 2014; see also Chiang \& Laughlin 2013). In addition, simulations that account for the effects of gas on protoplanet migration during the oligarchic growth phase find that systems are produced with planet masses decreasing steeply with orbital radius, which is different from the relatively flat scaling seen in STIPs (Ogihara et al. 2015).

The Inside-Out Planet Formation (IOPF) scenario proposed by Chatterjee \& Tan (2014, hereafter Paper I) is a new type of in situ formation model. It starts with pebble delivery to the disk midplane transition region between an innermost magnetorotational instability (MRI) active zone and a non-active "dead zone," where there is a local pressure maximum. The pebbles are trapped here and build up in a ring, which then forms a first protoplanet, perhaps involving a variety of processes including streaming (Youdin \& Goodman 2005) and gravitational (Toomre 1964) and/or Rossby wave (Varnière \& Tagger 2006) instabilities. The protoplanet is expected to continue its growth, especially by pebble accretion, until it becomes massive enough to open a gap in the disk. This gap, which may only be a partial gap with a modest reduction in gas mass surface density, pushes the pressure maximum outwards by at least a few Hill radii from the first planet, creating a new pebble trap, and thus the location of second planet formation (Hu et al. 2016, hereafter Paper III; see also Lambrechts et al. 2014). Furthermore, MRI activation in this gap region beyond the first planet may induce enhanced viscosity and thus a further outward retreat of the dead zone inner boundary (DZIB) 
pressure trap, leading to larger separations from the orbit of the second planet.

The pebble delivery rate is crucial for setting the timescale of pebble ring and planet formation in the IOPF scenario. To calculate this, we need to model the evolution of dust and pebbles in the protoplanetary disk and determine if the majority of solid material, which in the outer disk is initially in small dust grains, become incorporated into pebbles by the time it reaches the inner region. The IOPF model requires dust grains to grow into pebbles as smaller grains are well coupled to the gas and will not be trapped at a local pressure maximum (e.g., Zhu et al. 2012). The size distribution of pebbles is also important for models of planetesimal formation, e.g., via the streaming instability. This may be triggered in the pebble ring as a first step for in situ planet formation or in the outer disk as a potential first step for outer planet formation that may eventually truncate pebble delivery to the inner disk. Finally, modeling of dust and pebble evolution is a necessary step to provide observational predictions of the emission from the disk during various phases of IOPF.

There have been a number of previous studies of dust and pebble evolution in global disk models, including radial drift and particle interaction (e.g., Brauer et al. 2008; Birnstiel et al. 2010). Birnstiel et al. (2012) proposed a two-population model that divides dust into one smaller fixed-size group and one larger variable-size group that represents grain growth. Sato et al. (2015) proposed a simplified pebble-pebble interaction scenario by reducing the full Smoluchowski equation to a single-size evolution equation, yielding pebble fluxes and typical sizes in disks on scales down to $\sim 1$ au. A Lagrangian dust evolution model has been investigated by Krijt et al. (2016), which tracks batches of particles in the disk as they drift radially and grow through collisions.

In this paper, we present a new pebble-dust evolution model and apply it specifically to calculate the timescales needed to build up an STIP forming via IOPF, i.e., limited by the rate of pebble delivery. In Section 2, we review the equations that govern the disk models that are relevant to IOPF, including presenting new disk structure models that include realistic opacities and the transition from an "active," accretionpowered inner disk to a "passive," stellar-heated outer disk. In Section 3, we discuss a simple estimate of the timescale of the first, so-called "Vulcan" planet formation in steady accretion rate disks, including a reanalysis of the gap-opening mass criterion that was studied in Paper III. We also estimate the size of the region of the disk that is needed to supply the material for this planet. In Section 4, we model the radial drift and sweep-up growth of a single pebble in our model disks. In Section 5, we introduce our full pebble evolution model and numerical results for different disk parameters. In Section 6, we outline the assembly of an STIP-like system with pebble flux and planet mass criterion derived from earlier sections. We discuss the implications of our results and summarize our conclusions in Section 7.

\section{Disk Structure}

The structure of a steady accretion disk can be specified with a given accretion rate $\dot{m}$, Shakura \& Sunyaev (1973) viscosity parameter $\alpha$, stellar mass $m_{*}$, and midplane temperature versus radius relation $T(r)$ (in particular specifying whether the energy source of the disk is heating mostly by its own accretion, i.e., an "active" disk, or whether it is heating mostly by stellar irradiation, i.e., a "passive" disk):

$$
\begin{gathered}
T=T(r), \\
c_{s}=\left(\gamma k_{\mathrm{B}} T / \mu\right)^{1 / 2}, \\
h / r=c_{s} / \nu_{K}, \\
\nu=\alpha c_{s} h, \\
\Sigma_{g}=\dot{m} /(3 \pi \nu), \\
\rho=\Sigma_{g} /(h \sqrt{2 \pi}),
\end{gathered}
$$

where $c_{s}$ is the midplane sound speed, $\gamma$ is the power-law exponent of the barotropic equation of state $P=K \rho^{\gamma}, k_{\mathrm{B}}$ is Boltzmann's constant, $\mu=2.33 m_{\mathrm{H}}=3.90 \times 10^{-24} \mathrm{~g}$ is the mean particle mass (with the fiducial value set by assuming $\left.n_{\mathrm{He}}=0.2 n_{\mathrm{H} 2}\right), h$ is the disk vertical scale height, $v_{K}$ is the local Keplerian speed, $\nu$ is the viscosity, $\alpha \equiv 10^{-4} \alpha_{-4}$ is the Shakura-Sunyaev dimensionless viscosity parameter (with the fiducial value set for dead zone conditions, but we will consider other values also), $\Sigma_{g}$ is the gas mass surface density, $\dot{m} \equiv \dot{m}_{-9} 10^{-9} M_{\odot} \mathrm{yr}^{-1}$ is the accretion rate (with the fiducial normalization set by the observed accretion rates of transition disks, but again we will consider a range of values; see Paper I) and $\rho$ is the midplane gas density. Only Equations (1) and (2) depend on the energy source and equation of state, while the rest are general equations followed by all viscous disks.

The solution for the structure of an active accretion disk is achieved by balancing viscous-thermal dissipation with vertical radiative cooling (per unit area from one face of the disk):

$$
\frac{\nu \Sigma_{g} r^{2}}{2}\left(\frac{d \Omega}{d r}\right)^{2}=\frac{16 \sigma_{\mathrm{SB}}}{3 \Sigma_{g} \kappa} T^{4} .
$$

This generally applies in the inner disk. In this case, the temperature equation is (Paper I)

$$
\begin{aligned}
T= & \frac{3^{1 / 5}}{2^{7 / 5} \pi^{2 / 5}}\left(\frac{\mu}{\gamma k_{\mathrm{B}}}\right)^{1 / 5}\left(\frac{\kappa}{\sigma_{\mathrm{SB}}}\right)^{1 / 5} \\
& \times \alpha^{-1 / 5}\left(G m_{*}\right)^{3 / 10}\left(f_{r} \dot{m}\right)^{2 / 5} r^{-9 / 10}, \\
\rightarrow & 290 \gamma_{1.4}^{-1 / 5} \kappa_{10}^{1 / 5} \alpha_{-4}^{-1 / 5} m_{*, 1}^{3 / 10}\left(f_{r} \dot{m}_{-9}\right)^{2 / 5} r_{\mathrm{au}}^{-9 / 10} \mathrm{~K}
\end{aligned}
$$

and the gas mass surface density is given by

$$
\begin{aligned}
\Sigma_{g}= & \frac{2^{7 / 5}}{3^{6 / 5} \pi^{3 / 5}}\left(\frac{\mu}{\gamma k_{\mathrm{B}}}\right)^{4 / 5}\left(\frac{\kappa}{\sigma_{\mathrm{SB}}}\right)^{-1 / 5} \\
& \times \alpha^{-4 / 5}\left(G m_{*}\right)^{1 / 5}\left(f_{r} \dot{m}\right)^{3 / 5} r^{-3 / 5}, \\
\rightarrow & 812 \gamma_{1.4}^{-4 / 5} \kappa_{10}^{-1 / 5} \alpha_{-4}^{-4 / 5} m_{*, 1}^{1 / 5}\left(f_{r} \dot{m}_{-9}\right)^{3 / 5} r_{\mathrm{au}}{ }^{-3 / 5} \mathrm{~g} \mathrm{~cm}^{-2},
\end{aligned}
$$

where $\gamma \equiv 1.4 \gamma_{1.4}$ with fiducial normalization to a value of 1.4 for $\mathrm{H}_{2}$ with rotational modes excited, $\kappa \equiv \kappa_{10} 10 \mathrm{~cm}^{2} \mathrm{~g}^{-1}$ is the disk mean opacity (with the fiducial normalization here appropriate for inner disk conditions; but, note that we will more generally use tabulated opacities from Zhu et al. 2009), $\sigma_{\mathrm{SB}}$ is the Stefan-Boltzmann constant, $m_{*} \equiv m_{*, 1} M_{\odot}$ is the stellar mass with the fiducial normalization of $1 M_{\odot}$, $f_{r} \equiv 1-\sqrt{r_{*} / r}, r_{*}$ is the stellar radius, and $r_{\mathrm{au}} \equiv r /(1 \mathrm{au})$. The two parameters with some of the largest uncertainties are $\alpha$ 

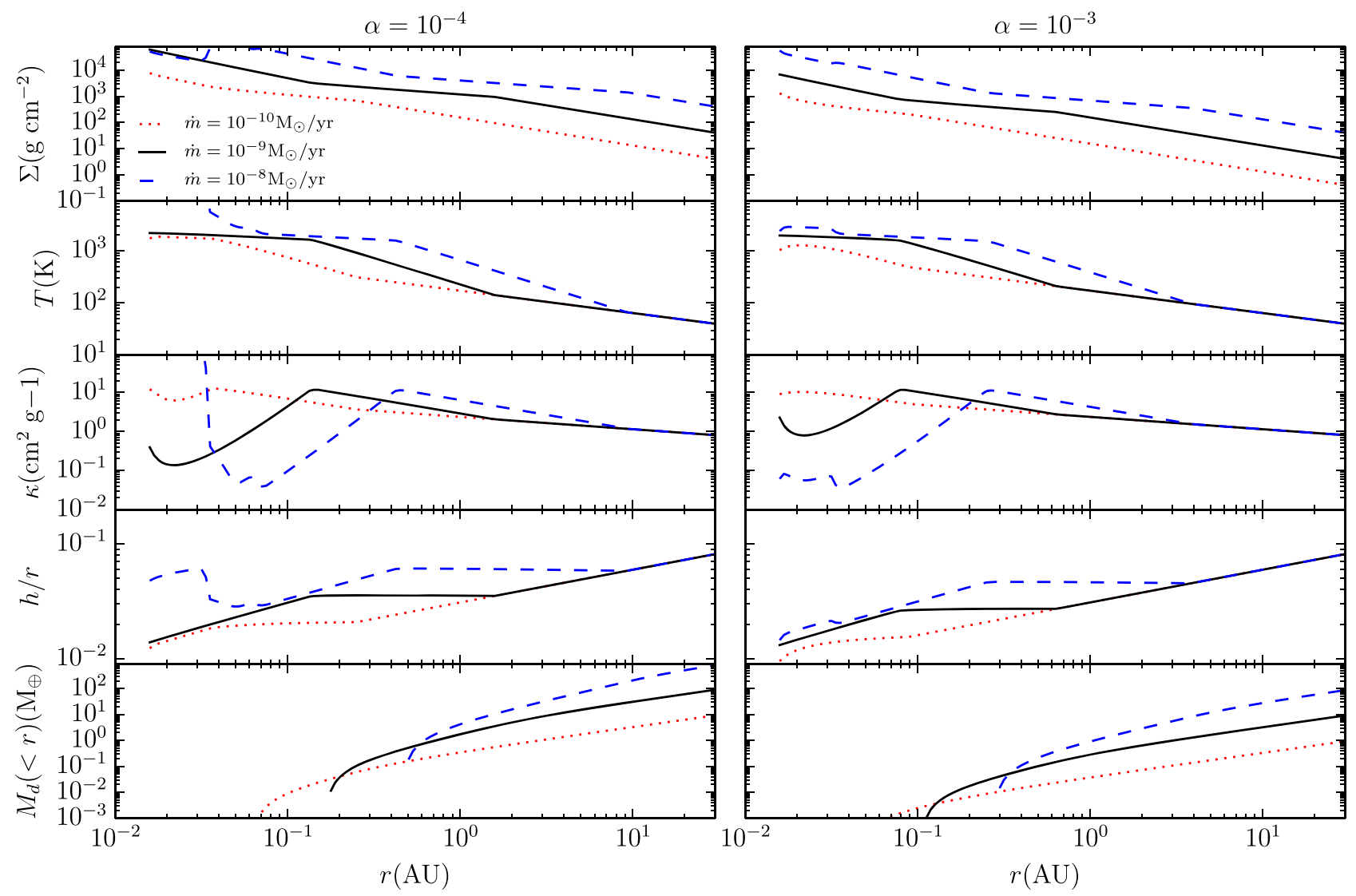

Figure 1. Structural profiles of the IOPF disk models used in this paper, assuming $\alpha=10^{-4}$ (left column) and $\alpha=10^{-3}$ (right column), for accretion rates $10^{-10}$ (red dotted), $10^{-9}$ (black solid), and $10^{-8} M_{\odot} \mathrm{yr}^{-1}$ (blue dashed). All models are for a one solar-mass central star. From top to bottom, the rows show gas mass surface density $\left(\Sigma_{g}\right)$, midplane temperature $(T)$, midplane opacity $\left(\kappa\right.$; assumed to be vertically constant), disk aspect ratio $(h / r)$, and the enclosed mass in solids $\left(M_{d}(<r)\right)$, i.e., it is initially assumed to be dust, summed in the disk from $r_{1200 \mathrm{~K}}$ out to a radius $r$ for a solid to gas mass ratio of $f_{s}=0.01$. Note that the model assumption of the disk vertical optical depth being $\gtrsim 1$ breaks down in the cases with $\alpha=10^{-3}$ and $\dot{m} \leqslant 10^{-9} M_{\odot} \mathrm{yr}^{-1}$ in the outer disk, e.g., for $\dot{m}=10^{-9} M_{\odot} \mathrm{yr}^{-1}$, this occurs at $r>8.5$ au.

and $\dot{m}$, and so we shall consider the effects of varying their fiducial values.

In the outer disk, stellar irradiation is expected to become the dominant energy source compared to local viscous accretion heating. Following Chiang \& Goldreich (1997), the temperature structure of a stellar irradiated, passive disk follows $T \propto r^{-3 / 7}$, which is much shallower than the $T \propto r^{-9 / 10}$ relation of the active disk.

To derive $T(r)$ in the passive disk regime, the following equations are solved iteratively:

$$
\begin{gathered}
T=(\beta / 4)^{1 / 4}\left(r_{*} / r\right)^{1 / 2} T_{*, \mathrm{eff}} ; \\
\beta=0.4 r_{*} / r+\eta H / r .
\end{gathered}
$$

Here, $\beta$ is the "grazing angle," i.e., the angle of incidence of stellar radiation at the disk surface, $T_{* \text {,eff }}$ is the surface photospheric temperature of the star, $\eta=2 / 7$ is the flaring index, i.e., $\eta=d(h / r) / d r$, and $H$ is the height of the disk photosphere above the midplane. Usually, $H=4 h$ is a good approximation in most parts of the disk (Chiang \& Goldreich 1997). If we choose a solar-mass T-Tauri star with temperature $T_{* \text {,eff }}=4500 \mathrm{~K}$ and radius $r_{*}=3.0 R_{\odot}=0.014$ au, we have the disk temperature versus radius equation

$$
T=172 r_{\mathrm{au}}^{-3 / 7} \mathrm{~K},
$$

which is independent of $\alpha$ and $\dot{m}$.
For our full numerical solutions, we set up a hybrid disk that is solved first in its inner region as an active disk, and then calculate a transition radius where the contribution from stellar irradiation becomes equal to that due to accretion heating. Beyond this transition radius, we scale the temperature in a manner appropriate for the passive disk regime, i.e., in particular, we compare the midplane temperatures of the active and passive disk models and choose the hotter one as the actual value.

The results of these constant $\alpha=10^{-4}$ (the fiducial value) and $10^{-3}$ and constant $\dot{m}=10^{-10}, 10^{-9}$, and $10^{-8} M_{\odot} \mathrm{yr}^{-1}$ disk models are shown in Figure 1. Such values of $\alpha$ have been inferred in the inner regions of simulated dead zones (Dzyurkevich et al. 2010). Whether such dead zone conditions and values of $\alpha$ continue to apply in larger-scale regions of the disk remains an open question (e.g., Mohanty et al. 2013); however, for simplicity we will assume that they do and calculate the structure of the disk out to $30 \mathrm{au}$. The range of accretion rates that we consider is guided by observations of transition disks (e.g., Manara et al. 2014). Another risk of applying a constant $\alpha$ and stable accreting disk to larger radius is overestimating the disk mass. In our fiducial case, as seen in Figure 1 , the dust mass within 30 au is about $100 M_{\oplus}$, while recent observations of dust continuum (van der Marel et al. 2016) give an average around $40 M_{\oplus}$ for most transitional disks. This factor of 2 should be considered when analyzing the simulation result. The active to passive transition radius for the 
fiducial disk $\left(\dot{m}=10^{-9} M_{\odot} \mathrm{yr}^{-1}, \alpha=10^{-4}\right)$ is 1.6 au. We note that the model assumption of the disk vertical optical depth being $\gtrsim 1$ breaks down in the cases with $\alpha=10^{-3}$ and $\dot{m} \leqslant 10^{-9} M_{\odot} \mathrm{yr}^{-1}$ in the outer disk, e.g., for $\dot{m}=$ $10^{-9} M_{\odot} \mathrm{yr}^{-1}$, this occurs for $r>8.5 \mathrm{au}$. Note also that an "inner transition" in the disk structure at 0.14 au results from the drop in opacity due to dust sublimation. However, in the context of IOPF, we expect $\alpha$ to also rise dramatically when $T \sim 1200 \mathrm{~K}$ (this location is referred to as $r_{1200 \mathrm{~K}}$ hereafter) as the MRI is activated by thermal ionization of alkali metals. This change in $\alpha$ has not been included in the models shown in Figure 1 since our main focus here is modeling the region that is the supply reservoir for solid material delivered to $\sim 0.1$ au locations. Still, we have extended the disk models within $0.1 \mathrm{au}$, since for the low accretion rate case $(\dot{m}=$ $\left.10^{-10} M_{\odot} \mathrm{yr}^{-1}\right), r_{1200 \mathrm{~K}}<0.1$ au.

In fact, there are some close-in planets with orbits within 0.1 au, e.g., Kepler-10b (Dumusque et al. 2014) is a superEarth planet orbiting a solar-type star at 0.016 au. For such planets to form in situ places constraints on IOPF models. For example, such systems would need to form in a disk with a relatively low accretion rate that has a small value of $r_{1200 \mathrm{~K}}$ (see below).

\section{3. "Vulcan" Planet Formation}

Our goal in this section is to obtain a simple estimate for the formation time and the size of the supply reservoir of the first, i.e., innermost planet to form in the IOPF scenario (Chatterjee \& Tan 2015, hereafter Paper II). To do this requires knowing the mass of such planets and the mass flux of pebbles. Here we will assume a steady accretion rate with most mass flux of solids being in the form of pebbles. The validity of this assumption will be explored in subsequent sections of the paper.

\subsection{Location}

The radial location of Vulcan planet formation is assumed to be set where the temperature reaches $\sim 1200 \mathrm{~K}$, which leads to the thermal ionization of alkali metals, especially $\mathrm{Na}$ and $\mathrm{K}$. This location varies depending on the accretion rate and other disk properties. In an active accretion disk, which is the relevant regime for the optically thick inner disk, this location is (Paper I)

$$
r_{1200 \mathrm{~K}}=0.218 \phi_{\text {DZIB }} \gamma_{1.4}^{-2 / 9} \kappa_{10}^{2 / 9} \alpha_{-4}^{-2 / 9} m_{*, 1}^{1 / 3}\left(f_{r} \dot{m}_{-9}\right)^{4 / 9} \mathrm{au},
$$

where $\phi_{\text {DZIB }}$ is a dimensionless parameter of order unity accounting for potential differences from a pure viscous disk model, e.g., due to the effects of extra energy extraction on the midplane temperature by a disk wind. Papers I and II adopted a fiducial value of $\phi_{\text {DZIB }}$ of 0.5 . In Paper III, we used 0.1 au as the typical location of Vulcan planets for hydrodynamic simulations of gap opening, where $\alpha=10^{-3}, \dot{m}=$ $10^{-9} M_{\odot} \mathrm{yr}^{-1}$, which corresponds to $\phi_{\text {DZIB }}=0.76$.

\subsection{Mass}

The mass of the Vulcan planet (and of all planets forming via IOPF) is assumed to be set by the gap opening, which then leads to the displacement of the local pressure maximum away from the planet to a larger radius in the disk, and thus the truncation of pebble accretion. Paper III investigated this process with $2 \mathrm{D}$ hydrodynamic simulations for the case of the fiducial accretion rate $\left(10^{-9} M_{\odot} \mathrm{yr}^{-1}\right)$, a value of $\alpha=10^{-3}$ for the inner dead zone that then rises to $\alpha=10^{-2}$ in the MRIactive region, and for the planet set at a fixed location of $0.1 \mathrm{au}$ from a $1 M_{\odot}$ star. The mass of the planet leading to the first significant displacement of the pressure maximum was assessed relative to the viscous-thermal criterion gap-opening mass of Lin \& Papaloizou (1993),

$$
\begin{aligned}
M_{G}= & \phi_{G} \frac{40 \nu m_{*}}{r^{2} \Omega_{K}} \\
= & 40 \phi_{G}\left(\frac{3}{128}\right)^{1 / 5} \pi^{-2 / 5}\left(\frac{\boldsymbol{\mu}}{\boldsymbol{k}_{\mathrm{B}}}\right)^{-4 / 5} \gamma^{4 / 5} \sigma_{\mathrm{SB}}^{-1 / 5} \\
& \times \alpha^{4 / 5} G^{-7 / 10} m_{*}^{3 / 10} \kappa^{1 / 5}\left(f_{r} \dot{m}\right)^{2 / 5} r^{1 / 10} \\
\rightarrow & 11.2 \phi_{G} \gamma_{1.4}^{4 / 5} \kappa_{10}^{1 / 5} \alpha_{-3}^{4 / 5} m_{*, 1}^{3 / 10}\left(f_{r} \dot{m}_{-9}\right)^{2 / 5} r_{0.1 \mathrm{au}}^{1 / 10} M_{\oplus} \\
\rightarrow & 1.77 \phi_{G} \gamma_{1.4}^{4 / 5} \kappa_{10}^{1 / 5} \alpha_{-4}^{4 / 5} m_{*, 1}^{3 / 10}\left(f_{r} \dot{m}_{-9}\right)^{2 / 5} r_{0.1 \mathrm{au}}^{1 / 10} M_{\oplus} .
\end{aligned}
$$

Here, $r_{0.1 \text { au }} \equiv r /(0.1 \mathrm{au})$. The last two numerical evaluations of this mass illustrate its relatively strong dependence on $\alpha$. In Paper III, we found that for the $\alpha=10^{-3}$ case, the critical pressure-displacement gap-opening mass was about $50 \%$ of the viscous-thermal gap-opening mass (i.e., this fraction was expressed as $\left.\phi_{G} \simeq 0.5\right)$. Note that the mass evaluated in Equation (14) is assessed only from the viscous criterion, but, for the conditions with $\alpha=10^{-3}$, it is also consistent with the thermal criterion, i.e., the Hill radius of the planet is similar in size to the disk scale height. However, as we see now, this condition breaks down when $\alpha=10^{-4}$, as the planet mass is much lower.

To better capture the behavior of gap opening for more general conditions in which the thermal criterion also begins to limit gap opening, we now improve our treatment by adopting the expression of Duffell (2015) for the gap-opening mass (the same scalings of $h / r$ and $\alpha$ is also presented in Zhu et al. 2013 and Fung et al. 2014):

$$
\begin{aligned}
M_{\mathrm{G}, \mathrm{D}}= & \phi_{\mathrm{G}, \mathrm{D}} \sqrt{3 \pi} m_{*} \alpha^{1 / 2}(\boldsymbol{h} / \boldsymbol{r})^{5 / 2} \\
= & \phi_{\mathrm{G}, \mathrm{D}} \frac{3^{3 / 4}}{2^{7 / 4}}\left(\frac{\mu}{\gamma k_{\mathrm{B}}}\right)^{-1}\left(\frac{\kappa}{\sigma_{\mathrm{SB}}}\right)^{1 / 4} \alpha^{1 / 4} \\
& \times G^{-7 / 8} m_{*}^{1 / 8}\left(f_{r} \dot{m}\right)^{1 / 2} r^{1 / 8}, \\
\rightarrow & 2.68 \phi_{\mathrm{G}, \mathrm{D}} \gamma_{1.4} \kappa_{10}^{1 / 4} \alpha_{-4}^{1 / 4} m_{*, 1}^{1 / 8}\left(f_{r} \dot{m}_{-9}\right)^{1 / 2} r_{0.1 \mathrm{au}}^{1 / 8} M_{\oplus},
\end{aligned}
$$

where $\phi_{\mathrm{G}, \mathrm{D}}$ is a dimensionless parameter of order unity (note that this absorbs the dimensionless factor, $f_{0}$, introduced by Duffell 2015). Note that the scaling of this relation is slightly different from the pebble isolation mass in Lambrechts et al. (2014), which depends on $h / r$ but not on $\alpha$. However, a more recent prescription for the pebble isolation mass in Bitsch et al. (2018) also includes a dependence on $\alpha$. A major difference of our results from these other studies is that our planet gapopening mass measurements are done specifically at a region in the disk where there is a transition in the value of $\alpha$ that causes a strong change in $\Sigma$. On the other hand, the results of Lambrechts et al. (2014) and Bitsch et al. (2018) are for disks with constant $\alpha$ and thus a relatively slowly varying $\Sigma_{g}$ profile. 


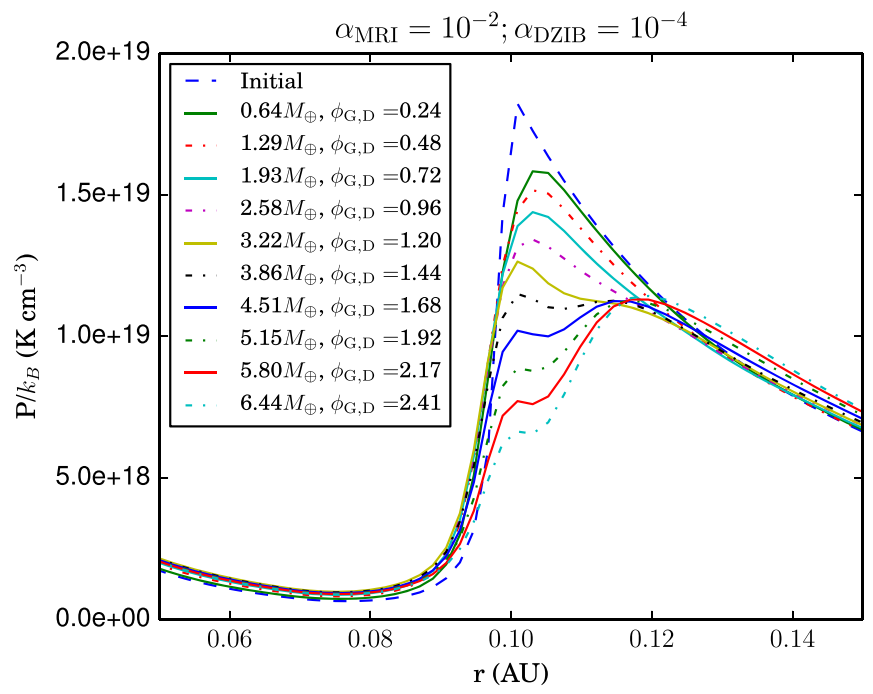

Figure 2. Steady-state, cylindrically averaged disk midplane pressure profiles, starting with an initial case with no planet. The pressure maximum, due to the dead zone inner boundary, has been set at 0.1 au. We set $\alpha=0.01$ in the inner MRI-active disk, transitioning to $10^{-4}$ in the dead zone. Then, the effects of adding planets of increasing mass and held fixed at 0.1 au are studied. These planets open gaps of increasing depth, which eventually leads to the displacement of the local pressure maximum away from the planet. This occurs for $M_{p}=3.86 M_{\oplus}$, i.e., $\phi_{\mathrm{G}, \mathrm{D}}=1.44$.

To test Equation (15) and constrain $\phi_{\mathrm{G}, \mathrm{D}}$, we now extend the simulations of Paper III to investigate the gap-opening mass for different values of $\alpha$ and $\dot{m}$. We perform a series of FARGO (Masset 2000) hydrodynamic simulations on disks with an $\alpha$ viscosity transition. The inner region is still assumed to be an MRI-active zone with $\alpha=0.01$. Beyond this is the dead zone with a much lower value of $\alpha$. We implement the transition following the same methods as in Paper III, with the transition set to be at $0.1 \mathrm{au}$, which is the location of the local pressure maximum before the introduction of a planet in the disk. Then, planets of different masses are inserted in the disk and held fixed at this location (note that Paper III showed that growing planets would be trapped here given the torques exerted by the disk). We study the cylindrically averaged pressure profiles and identify the critical gap-opening mass for IOPF as corresponding to the point where the local pressure maximum in the disk becomes significantly displaced from the planet, i.e., by more than its Hill radius,

$$
R_{\mathrm{H}} \equiv\left(\frac{M_{p}}{3 m_{*}}\right)^{1 / 3} r \rightarrow 1.00 \times 10^{-3} M_{p, \oplus}^{1 / 3} m_{*, 1}^{-1 / 3} r_{0.1 \mathrm{au}} \text { au }
$$

where $M_{p, \oplus} \equiv M_{p} / M_{\oplus}$ is the planet mass normalized to one Earth mass.

Figure 2 shows examples of these pressure profiles for the case of a disk with $\dot{m}=10^{-9} M_{\odot} \mathrm{yr}^{-1}$ and a transition from $\alpha=10^{-2}$ to $\alpha=10^{-4}$ in the vicinity of 0.1 au. We see in this case that a planet with a mass of $3.86 M_{\oplus}$ is able to open a gap deep enough that the local pressure maximum retreats significantly, i.e., by $\sim 0.015$ au (in this case, $\sim 10$ Hill radii), from the planet. This mass scale corresponds to a value of $\phi_{\mathrm{G}, \mathrm{D}}=1.44$ in the normalization of the Duffell (2015) gap-opening mass (Equation (15)). In the IOPF model, this means that the next planet may form from a new pebble ring at this location or somewhat farther out if the MRI-active region also spreads outwards in the lower densities enabled by the gap opening.
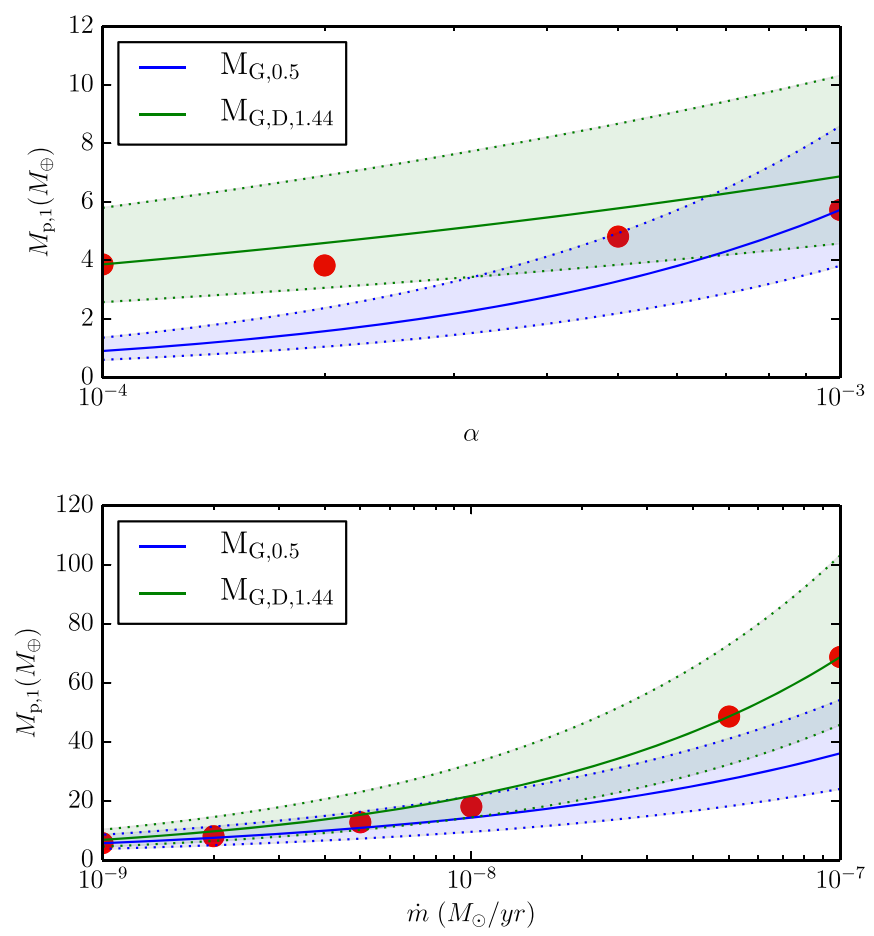

Figure 3. Planet gap-opening mass estimates based on the viscous criterion ( $M_{\mathrm{G}, 0.5}$; blue) and the Duffell (2015) criterion ( $M_{\mathrm{G}, \mathrm{D}}$; green) as a function of viscosity $\alpha$ (top panel) and accretion rate $\dot{m}$ (bottom panel). The shaded area indicates a range of a factor of 1.5 variation about the fiducial mass estimates. The red dots are the FARGO simulation results based on the local pressure maximum retreat. Note that the Duffell (2015) criterion is a better match with the simulation results in the low- $\alpha$ and high- $\dot{m}$ regimes.

Figure 3(a) shows the results of four different series of simulations, including the example shown in Figure 2, in which the $\alpha$ in the dead zone is varied from $10^{-4}$ to $10^{-3}$ and the gapopening masses (by the pressure maximum displacement criterion) are assessed and compared to those predicted by the simple viscous criterion (Equation (14)) and the Duffell (2015) estimate (Equation (15)). Figure 3(b) shows the results of a similar series of gap-opening simulations, but now varying $\dot{m}$ from $10^{-10}$ to $10^{-7} M_{\odot} \mathrm{yr}^{-1}$. We find that the simple viscous criterion underestimates the planet mass required to displace the pressure maximum in both the low- $\alpha$ and high- $\dot{m}$ regimes. These are the conditions when the disk is relatively denser and hotter, and its vertical scale height is relatively large compared to the Hill sphere of the planet. Our results are consistent with those of Duffell \& MacFadyen (2013): they discuss how gap profiles scale differently when a planet's Hill radius is smaller than the disk scale height. We note that our results are derived from 2D simulations, which should ideally be confirmed by $3 \mathrm{D}$ simulations that resolve the vertical structure of the disk to more accurately predict midplane pressure structures.

With our improved estimate of the Vulcan planet mass set by the gap opening (Equation (15)), following Paper II, we eliminate the accretion rate term, $f_{r} \dot{m}_{-9}$, by combining it with Equation (13). Previously, Paper II did this by using the viscous criterion gap-opening mass to obtain $M_{p, 1}=$ $0.83 \phi_{G, 0.5} \phi_{\text {DZIB }, 0.5}^{-9 / 10} \gamma_{1.4} \alpha_{-4} r_{0.1 \text { au }} M_{\oplus}$. Now, we find a revised mass versus orbital radius relation for Vulcans,

$$
\begin{aligned}
M_{p, 1}= & 3.50 \phi_{\mathrm{G}, \mathrm{D}, 1.44} \phi_{\mathrm{DZIB}, 0.5}^{-9 / 8} \gamma_{1.4}^{5 / 4} \\
& \times \alpha_{-4}^{1 / 2} m_{*, 1}^{-1 / 4} r_{0.1 \mathrm{au}}^{5 / 4} M_{\oplus} .
\end{aligned}
$$


Table 1

Pebble Supply Reservoir Radii and Drift Timescales for Vulcan Planets

\begin{tabular}{lccccc}
\hline \hline $\begin{array}{l}\text { Disk Model } \\
\left(\dot{m}_{-9}, \alpha\right)\end{array}$ & $\begin{array}{c}M_{p, 1} \\
\left(M_{\oplus}\right)\end{array}$ & $\begin{array}{c}r_{1200 \mathrm{~K}} \\
(\mathrm{au})\end{array}$ & $\begin{array}{c}r_{\text {res, }, 1}(\mathrm{au}) \\
\left(\epsilon_{p, 1}=1,0.5,0.25\right)\end{array}$ & $\begin{array}{c}t_{\text {drift }} \text { (no growth) } \\
\left(10^{4} \mathrm{yr}, \epsilon_{p, 1}=1,0.5,0.25\right)\end{array}$ & $\begin{array}{c}t_{\text {drift }}(w / \text { sweep-up growth }) \\
\left(10^{4} \mathrm{yr}, \epsilon_{p, 1}=1,0.5,0.25\right)\end{array}$ \\
\hline $0.1,10^{-4}$ & 0.96 & 0.060 & $3.35,6.87,14.3$ & $6.20,7.23,8.18$ & $0.748,1.17,1.71$ \\
$0.1,10^{-3}$ & 1.59 & 0.036 & $19.1,40.2,84.5$ & $0.994,1.22,1.94$ & $0.474,0.697,1.41$ \\
$1,10^{-4}$ & 3.43 & 0.167 & $1.75,2.95,5.45$ & $16.3,24.6,33.6$ & $0.798,1.35,2.35$ \\
$1,10^{-3}$ & 5.73 & 0.100 & $6.67,13.7,28.5$ & $5.43,6.37,7.23$ & $1.14,1.67,2.28$ \\
$10,10^{-4}$ & 12.3 & 0.464 & $1.93,2.90,4.47$ & $17.7,29.9,50.4$ & $0.671,1.14,2.08$ \\
$10,10^{-3}$ & 20.6 & 0.278 & $3.73,6.14,11.1$ & $12.6,19.8,27.7$ & $1.39,2.40,3.98$ \\
\hline
\end{tabular}

Note. $t_{\text {drift }}$ is evaluated for pebbles of initial radius $1 \mathrm{~mm}$, with the trajectory followed from $r_{\mathrm{res}, 1}$ to the DZIB, where $r=r_{1200 \mathrm{~K}}$ (for $\phi_{\mathrm{DZIB}}=0.76$ ).

We see that this has a slightly steeper scaling of $M_{p, 1} \propto r^{5 / 4}$ compared to the Paper II result. The implications of this and other revised IOPF predictions, such as the more general $M_{p}$ versus $r$ relation of Equation (15), for the comparison with observed STIPs will be examined in a future paper.

\subsection{Formation Timescale}

Now that we have improved estimates for the masses of Vulcan planets, we assess their formation timescales. Consider a protoplanetary disk with a mass accretion rate of $\dot{m}$ and solids to gas mass ratio of $f_{s}$. We assume $f_{s}=0.01$ as a fiducial value. The solids are divided into two parts: (1) submillimeter- or smaller-sized dust grains that are well coupled to the gas and (2) larger, typically centimeter-sized "pebbles" that can decouple from the gas flow and can be subject to substantial radial drift due to gas drag.

Pebbles are expected to arise from the coagulation of dust grains, mainly via Brownian motion and turbulent mixing. The maximum steady-state rate of pebble delivery to a particular radial location in the disk is when most solids are in the form of pebbles, i.e., $f_{p}=\dot{m}_{p} / \dot{m}_{s} \simeq 1$. The accretion-limited pebble supply rate is then simply

$$
\begin{aligned}
\dot{m}_{p} & =10^{-11} f_{p} f_{s, 0.01} \dot{m}_{-9} M_{\odot} \mathrm{yr}^{-1} \\
& =3.33 \times 10^{-6} f_{p} f_{s, 0.01} \dot{m}_{-9} M_{\oplus} \mathrm{yr}^{-1}
\end{aligned}
$$

Thus, we have an estimate of the planet formation time if limited by a steady accretion rate of pebbles,

$$
\begin{aligned}
t_{\mathrm{form}}= & M_{\mathrm{G}, \mathrm{D}, 1.44} / \dot{m}_{p} \\
= & f_{s}^{-1} f_{0}^{-1 / 2} \frac{3^{3 / 4}}{2^{7 / 4}}\left(\frac{\mu}{\gamma k_{\mathrm{B}}}\right)^{-1}\left(\frac{\kappa}{\sigma_{\mathrm{SB}}}\right)^{1 / 4} \alpha^{1 / 4} \\
& \times G^{-7 / 8} m_{*}^{1 / 8} f_{r}^{1 / 2} \dot{m}^{-1 / 2} r^{1 / 8}, \\
\rightarrow & 1.16 \phi_{\mathrm{G}, \mathrm{D}, 1.44} f_{s,-2}^{-1} \\
& \times \gamma_{1.4} \kappa_{10}^{1 / 4} \alpha_{-4}^{1 / 4} m_{*, 1}^{1 / 8} f_{r}^{1 / 2} \dot{m}_{-9}^{-1 / 2} r_{0.1 \mathrm{au}}{ }^{1 / 8} \mathrm{Myr} .
\end{aligned}
$$

For solar-type stars, estimates of the lifetimes of protoplanetary disks are $\sim 1$ to $10 \mathrm{Myr}$, with median values of $\sim 3 \mathrm{Myr}$, (e.g., Williams \& Cieza 2011; Ribas et al. 2015). Since there are usually $\gtrsim 3$ planets in a typical STIP, this $\sim 1$ Myr formation time for the first planet may give an interesting constraint on IOPF models: e.g., to form $\sim 3$ planets may take a timescale that is very similar to typical disk lifetimes. Note that the above planet-formation timescale is not very sensitive to model parameters, such as opacity $(\kappa)$, viscosity $(\alpha)$, and location $(r)$.

\subsection{Pebble Supply Reservoir}

The radius of the "pebble supply reservoir," $r_{\text {res, } 1}$, that is needed to construct a Vulcan planet can now also be evaluated by equating this to the disk radius that encloses a solid mass that is at least as large as $M_{p, 1}$. We define

$$
M_{p, 1}=\epsilon_{p, 1} M_{d}\left(<r_{\mathrm{res}, 1}\right),
$$

and show some example estimates of $r_{\text {res, } 1}$ in Table 1, given our disk models (e.g., those shown in Figure 1). Here, $\epsilon_{p, 1}$ denotes planet-formation efficiency, i.e., the fraction of solids within the reservoir that finally becomes part of the planet. The size of the supply reservoir is sensitive to the value of $\alpha$ adopted for the disk model. For the fiducial case of $\alpha=10^{-4}$ (i.e., potentially appropriate for dead zone conditions) and with $\dot{m}=10^{-9} M_{\odot} \mathrm{yr}^{-1}$, we find $r_{\text {res, } 1} \simeq 3 \mathrm{au}$. However, if the value of $\alpha$ is larger, then $r_{\text {res, } 1}$ grows, potentially to several tens of astronomical units, both because of the larger planet mass and the lower-mass surface density of the disk.

\section{Single Pebble Evolution Model}

Here, we describe a model of single pebble evolution in the disk, i.e., involving radial drift due to gas drag and growth of the pebble through sweeping up small dust grains. This model is the basis of that used in the next section to predict the global evolution of the pebble population. Here we will first use the single pebble evolution model for simple estimates of the supply timescales needed to form Vulcan planets.

The pebble evolution model is based on that presented by $\mathrm{Hu}$ et al. (2014). It includes four different drag regimes (Epstein and three Stokes regimes, depending on the relative radius of the pebble, $a_{p}$ to the mean free path for collisions in the gas, $\lambda$, and the Reynolds number, $R e$ ) to evaluate the drag force $F_{D}$. We then calculate the gas drag frictional timescale of a pebble of mass $m_{p}$ moving at speed $v_{p}$ relative to gas as $t_{\text {fric }}=\left(m_{p} v_{p}\right) / F_{D}$,

$$
t_{\text {fric }}= \begin{cases}\rho_{p} a_{p} /\left(\rho v_{p}\right) & \text { if } a_{p}<9 \lambda / 4 \\ 2 \rho_{p} a_{p}^{2} /(9 \nu \rho) & \text { if } a_{p}>9 \lambda / 4 \& \operatorname{Re}<1 \\ \left(\rho_{p} a_{p} /\left[9 \rho v_{p}\right]\right)\left(2 a v_{p} / \nu\right)^{0.6} & \text { if } 1<\operatorname{Re}<800 \\ 8 \rho_{p} a_{p} /\left(1.32 \rho v_{p}\right) & \text { if } \operatorname{Re}>800 .\end{cases}
$$

Then the radial drift of the pebble relative to gas is (Armitage 2007)

$$
v_{r, p} \simeq-k_{P}\left(c_{s} / v_{K}\right)^{2}\left(\tau_{\text {fric }}+\tau_{\text {fric }}^{-1}\right)^{-1} v_{K},
$$




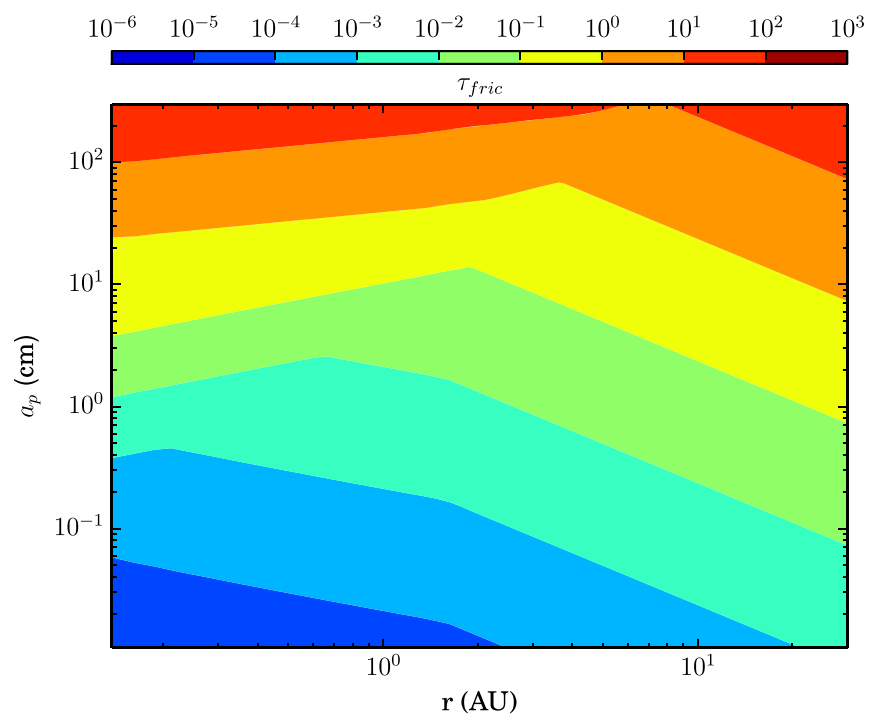

Figure 4. Dimensionless friction timescale, $\tau_{\text {fric }}$, map of pebbles in our fiducial disk model $\left(\dot{m}=10^{-9} M_{\odot} \mathrm{yr}^{-1}, \alpha=10^{-4}\right)$.

where $k_{P}$ is the power-law index of the pressure-radius relation in $P=P_{0}\left(r / r_{0}\right)^{-k_{P}}, \tau_{\text {fric }} \equiv \Omega_{K} t_{\text {fric }}$ is the dimensionless friction timescale, and $\Omega_{K}$ is the orbital angular frequency. An example of the values of $\tau_{\text {fric }}$ as a function of pebble size and disk radius for our fiducial disk model ( $\dot{m}=10^{-9} M_{\odot} \mathrm{yr}^{-1}, \alpha=10^{-4}$ ) is shown in Figure 4. We can see from this figure that efficient radial drift, i.e., when $\tau_{\text {fric }} \sim 1$, occurs for $\sim$ centimeter-sized pebbles in the outer disk region, which will be important for the models we consider below that are based on a steady injection of pebbles at this outer disk scale (see Section 5).

The pebble evolution model of $\mathrm{Hu}$ et al. (2014) involves the "sweep-up growth" of pebbles through the accretion of small dust grains (we add pebble-pebble coagulation in the next section). Similar models have been proposed by Safronov (1972) and also been discussed by Dullemond \& Dominik (2005) in the context of dust growth during vertical settling. Windmark et al. (2012) considered such a model as a mechanism by which centimeter-sized "seeds" can overcome the bouncing/fragmentation barrier to form planetesimals by sweeping up smaller grains.

Like Hu et al. (2014), we assume a pebble sweeps up all of the dust within its geometric cross-section if it is in the Epstein drag regime, where the pebble size is comparable to a gas molecule's mean free path. However, when a pebble enters the Stokes regime, the gas behaves more like a continuous fluid, forming a pressure wake in front of the pebble. We thus assume sweep-up growth stops, as small dust grains are deflected by this pressure wake and flow away following gas streamlines.

To implement this model, in each time step $\delta t$, the mass growth of a pebble is

$$
\delta m_{p}=\pi a_{p}^{2} v_{\text {rel }} \rho_{d} \delta t .
$$

Here, $\rho_{d}$ is the mass of dust per unit volume in the disk, i.e., $1 \%$ of the gas density, $\rho$, for our fiducial initial conditions. $v_{\text {rel }}$ is the relative velocity between a pebble and its surrounding gas, which is given by

$$
v_{\text {rel }}=\left(1+\tau_{\text {fric }}^{2} / 4\right)^{1 / 2} v_{r, p} .
$$

We now calculate example trajectories of pebbles of initial radius of $1 \mathrm{~mm}$ that start at a radius in the disk equal to that of the pebble supply reservoir for a Vulcan planet, $r_{\text {res,1 }}$ (see Figure 5). The trajectories are shown for the cases with and without sweep-up growth, i.e., the latter being for pebbles of constant radius of $1 \mathrm{~mm}$. These calculations are done for six different disk models, i.e., $\dot{m}_{-9}=0.1,1$, and 10 and $\alpha=10^{-4}$ and $10^{-3}$. In each disk, three different starting radii for $\epsilon_{p, 1}=1,0.5$, and 0.25 are considered. These properties and the total drift times, $t_{\text {drift }}$, for all of these cases are listed in Table 1.

Figure 5 and Table 1 illustrate several points. First, note the dependence of the Vulcan planet masses, $M_{p, 1}$, and the pebble supply reservoir outer radii, $r_{\text {res, } 1}$, on disk properties. These planet masses range from about $1 M_{\oplus}$ in the case of a low accretion rate, low viscosity disk, to about $20 M_{\oplus}$ in a disk with a $100 \times$ higher accretion rate and $10 \times$ higher viscosity. Since disk mass surface densities are lower for higher viscosity disks, the corresponding reservoir radii also become larger in such cases. Thus, $r_{\text {res, } 1}$ varies from just 1.75 au (actually occurring in the fiducial case if $\epsilon_{p, 1}=1$ ) to almost 100 au in the low- $\dot{m}$, high- $\alpha$, low- $\epsilon_{p, 1}$ model.

Then, the drift times from these radii to the location of planet formation, i.e., $r_{1200 \mathrm{~K}}$, depend on whether the pebble is allowed to grow through the sweep-up growth of small dust grains. Without growth, in the $\alpha=10^{-4}$ disks, we see that $t_{\text {drift }} \gtrsim 10^{5}$ yr. It takes longer to drift inwards in the high- $\dot{m}$ disks, even though starting closer in, because the pebbles are in different drag regimes leading to different drag frictional timescales (Equation (21)). This pattern is mirrored in the higher- $\alpha$ disks, where we see that the no-growth drift times can be as short as $\sim 10^{4} \mathrm{yr}$ even from $\sim 100$ au. Allowing pebbles to grow results in shorter drift timescales, since the pebbles approach sizes that lead to maximal drag force, i.e., minimal frictional timescales, leading to efficient radial migration, which then further enhances pebble growth. We notice that in all six disk models considered, the values of $t_{\text {drift }}$ with sweep-up growth are quite similar, i.e., $\sim 10^{4} \mathrm{yr}$. The pebbles can grow to sizes of $\sim 1-10 \mathrm{~cm}$.

Thus, we conclude that for a wide range of disk parameters $\left(\dot{m} \in\left[10^{-10}, 10^{-8}\right] M_{\odot} \mathrm{yr}^{-1}, \alpha \in\left[10^{-4}, 10^{-3}\right]\right)$, the radial drift timescale is one or two orders of magnitude shorter than $t_{\text {form }}$, based on an estimate of a steady-state supply rate of pebbles. This reflects the extreme limit of the pebble supply rate being boosted above the accretion-limited rate because of the net radial drift of pebbles with respect to gas. It also assumes that the pebbles starting from within $r_{\text {res, } 1}$ will be able to sweep up a large fraction of the total solid mass, i.e., of dust, in this inner disk region. To advance beyond these simple estimates, we need to construct a global model of pebble evolution and supply in the disk.

\section{Global Pebble Evolution Model}

\subsection{Numerical Implementation}

Here we describe the basic algorithm used in an Eulerian pebble evolution model, including radial drift and growth (sweep-up plus coagulation). We sample a discrete grid in radial distance $(r)$ and pebble radius $\left(a_{p}\right)$. At any given radius, the model is intended to approximate conditions applicable to pebbles drifting inwards near the disk midplane. 

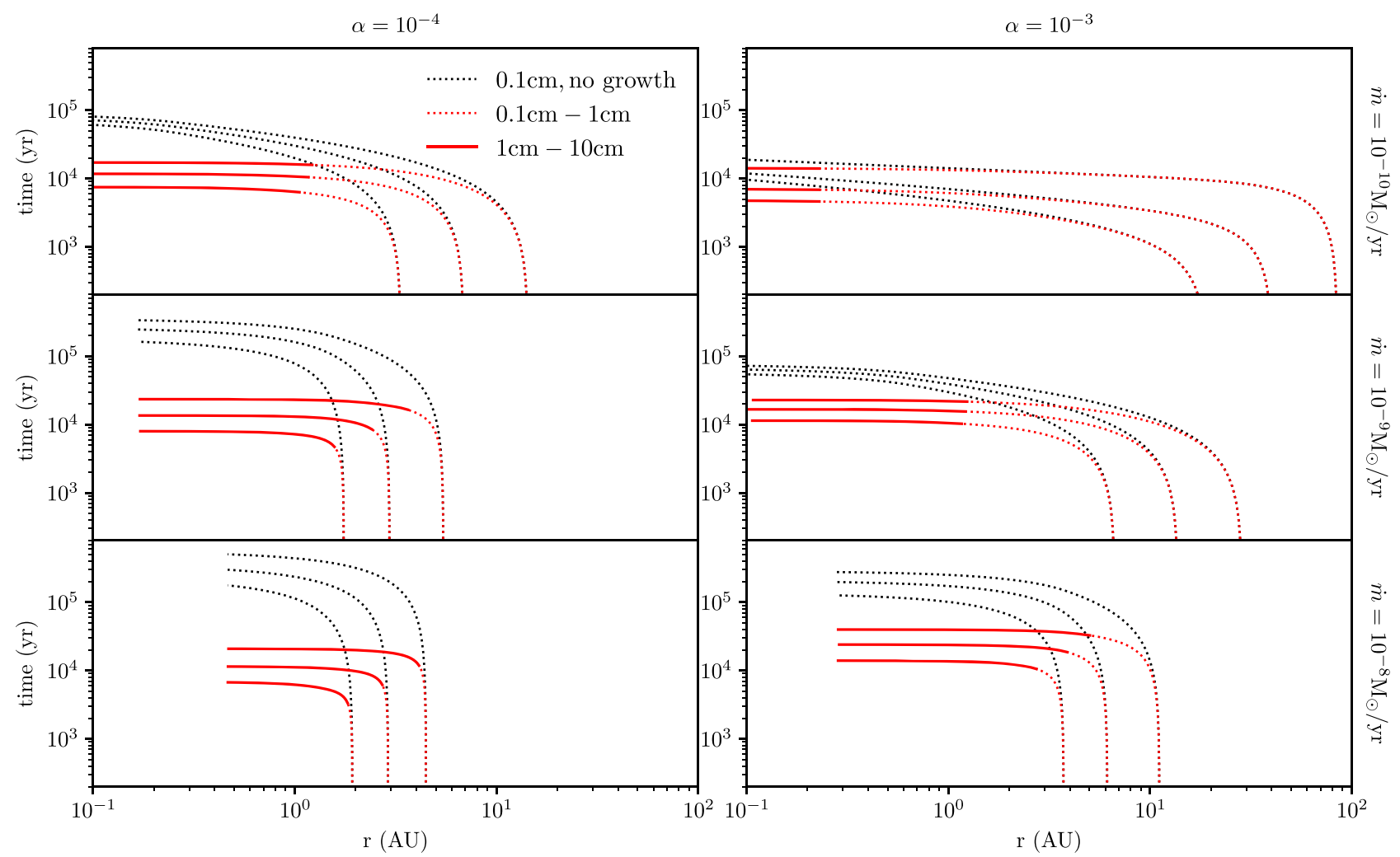

Figure 5. Radial trajectories in time of pebbles with initial radii of $0.1 \mathrm{~cm}$, starting from various supply reservoir outer boundaries for Vulcan planets with $\epsilon_{p}=1,0.5$, and 0.25 (see Table 1). The black dotted lines show models with no growth of the pebbles, i.e., they keep a constant radius of $0.1 \mathrm{~cm}$. The red lines show models with growth via Stokes-limited sweep-up of small dust grains. The different styles of the red lines represent different ranges of pebble sizes during this growth: dashed lines cover the size range of 0.1 to $1 \mathrm{~cm}$; solid lines cover 1 to $10 \mathrm{~cm}$. The left column shows disks with $\alpha=10^{-4}$, the right column those with $\alpha=10^{-3}$. The top, middle, and bottom rows show models with $\dot{m}=10^{-10}, 10^{-9}, 10^{-8} M_{\odot} \mathrm{yr}^{-1}$, respectively. Pebble trajectories are followed until an inner radius is reached, where $r=r_{1200} \mathrm{~K}$, $\phi_{\text {DZIB }}=0.76$, which is expected to be the location of the DZIB.

We divide solids in the disk into two groups: larger particles treated as pebbles and smaller particles treated as dust. Dust represents particles smaller than a certain size threshold, for which we adopt $a_{p, \min }=0.01 \mathrm{~cm}$ (as a fiducial choice), while pebbles are particles divided into different size bins. During radial drift, pebbles can grow by sweeping up dust (i.e., the Stokes-limited sweep-up growth model described in the previous section) and by coagulation with other pebbles.

The basic numerical approach in an "Eulerian" model is to find the fraction of material that is transported between neighboring cells within each time step. Considering pebble evolution, this fraction at radial grid cell $i$ and pebble-size grid cell $j, f_{p}(i, j)$, is calculated as the sum of the radial drift fraction in $r$ space, $f_{p, r}(i, j)$, and size $\left(a_{p}\right)$ space, $f_{p, a}(i, j)$. The radial drift fraction of mass moved to the next radial grid, i.e., the mass fraction of pebble size $j$ at radial grid $i, f_{p, r}(i, j)$, is the ratio between the radial drift distance to the radial width of the ring-shaped grid:

$$
f_{p, r}(i, j)=v_{r, i, j} \Delta t / \Delta r_{\text {ring }},
$$

where $\Delta r_{\text {ring }}$ is the width of each radial grid, $\Delta t$ is the time step, and $v_{r, i, j}$ is the radial drift velocity of pebble size $j$ at the center of the grid. This "center of box" first-order approximation works because the variation of disk properties within each grid is very minor, especially with a logarithmic radial grid set-up.

The size evolution of pebbles is more complicated. The difference between the largest and smallest pebbles within one size bin becomes larger due to the nature of sweep-up growth. At size bin $j$, the minimum size is denoted as $a_{p, i, j \min }$, and the maximum is $a_{p, i, j \max }$. Within one time step, a pebble with size $a_{p, i, j \min }$ can grow to size $a_{p, i, j \min }^{\prime}$ by sweeping up dust of mass $\delta m$ from Equation (23),

$$
a_{p, i, j \min }^{\prime}=\left(a_{p, i, j \min }^{3}+\frac{3 \delta m}{4 \pi}\right)^{1 / 3} .
$$

Similarly, a $a_{p, i, j \max }$-sized pebble can grow to $a_{p, i, j \max }^{\prime}$. Between size $a_{p, i, j}$ and $a_{p, i, j+1}$, there exists a size $a_{p, i, j \text { mid, }}$, that can grow to $a_{p, i, j \max }$ in this time step, $\Delta t$. The value of $a_{p, i, j}$ mid is obtained from the linear interpolation of $a_{p, i, j \text { min }}$ and $a_{p, i, j \max }$. Thus, pebbles below size $a_{p, i, j \text { mid }}$ will stay in the current size bin, while pebbles above $a_{p, i, j \text { mid }}$ move to the next size bin. With the assumption that pebble mass is distributed evenly within each size bin, we obtain the fractional mass in size space that grows to the next size bin,

$$
f_{p, a}(i, j)=\frac{\left(a_{p, i, j \max }-a_{p, i, j \operatorname{mid}}\right)}{\left(a_{p, i, j \max }-a_{p, i, j \min }\right)} .
$$

To make the code more efficient, at each mesh in $r$ space, the time step is self-adjustable, depending on the drift rate and growth rate of pebbles. The entire disk shares a larger time step for synchronization, while each mesh has its own sub-time step that is a simple fraction $(1 / n)$ of the larger step. To achieve this, virtual rings are implemented between each mesh, recording fluxes from the outer mesh and supplying them to inner meshes in each substep. 


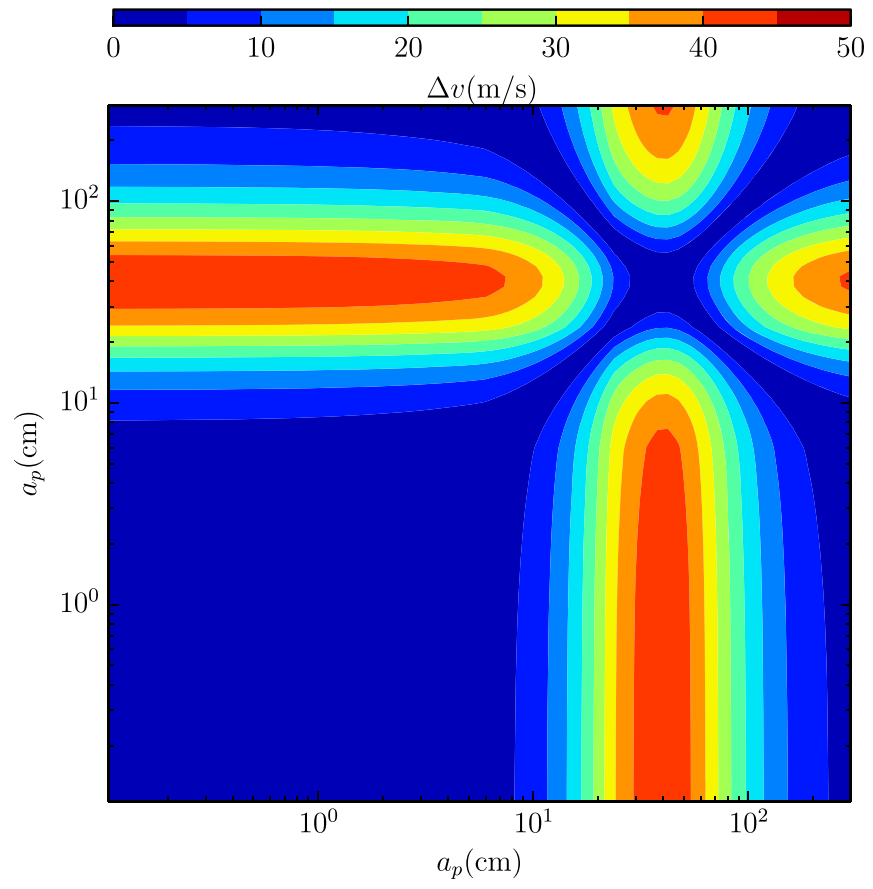

Figure 6. Example of total disk plane relative velocities between different pebbles due to radial and cylindrical drift in the fiducial disk model $\left(\dot{m}_{-9}=1\right.$, $\alpha=10^{-4}$ ) at $1 \mathrm{au}$. These relative velocities are used in the pebble-pebble coagulation model.

A simple pebble-pebble interaction algorithm is also implemented. Here we ignore effects like Brownian motion, disk turbulence, or vertical settling, so that only radial and cylindrical drifts are considered for setting interaction velocities. When pebble-size group $j$ meets group $k$, the number of two-body interactions leading to coagulation, $N_{c}$, per unit area per time step, $\Delta t$, is calculated as (see Birnstiel et al. 2010)

$$
N_{c}=S_{j, k} \Delta v_{j, k} \Delta t p_{c} N_{j} N_{k} / \sqrt{2 \pi\left(h_{j}^{2}+h_{k}^{2}\right)},
$$

where $S_{j, k}=\pi\left(a_{p, j}+a_{p, k}\right)^{2}$ is the coagulation cross-section, $\Delta v_{j, k}$ is the relative velocity between pebble group $j$ and group $k, N_{j}$ and $N_{k}$ are the surface number densities of the pebbles in these groups, $h_{j}$ and $h_{k}$ are the pebble vertical scale heights, calculated as $h_{j}=h / \sqrt{1+\tau_{\text {fric }, j} / \alpha}$, and $p_{c}$ is the coagulation efficiency given by

$$
p_{c}= \begin{cases}1, & \text { if } \Delta v \leqslant 10 \mathrm{~m} \mathrm{~s}^{-1} \\ 3-\left(\Delta v / 5 \mathrm{~m} \mathrm{~s}^{-1}\right), & \text { if } 10 \mathrm{~m} \mathrm{~s}^{-1}<\Delta v \leqslant 15 \mathrm{~m} \mathrm{~s}^{-1} \\ 0, & \text { if } \Delta v \geqslant 15 \mathrm{~m} \mathrm{~s}^{-1}\end{cases}
$$

Thus, when the relative speed is $>15 \mathrm{~m} \mathrm{~s}^{-1}$, there is a "fragmentation barrier" that prevents further pebble growth.

Figure 6 shows an example of the relative velocities occurring between different size groups at a 1 au location in the fiducial disk with $\dot{m}=10^{-9} M_{\odot} \mathrm{yr}^{-1}$ and $\alpha=10^{-4}$. These range up to $\sim 40 \mathrm{~m} \mathrm{~s}^{-1}$ for pebbles with $a_{p} \sim 40 \mathrm{~cm}$, but, for typical sizes of $\sim 1$ to $10 \mathrm{~cm}$, are $\lesssim 10 \mathrm{~m} \mathrm{~s}^{-1}$.

The final step of modeling coagulation is redistributing the masses of the coagulation products. Each pebble group has a certain range of sizes (i.e., masses), and the width of this range would be enlarged by coagulation, as the product's minimum size is set by the combination of the smallest pebbles from both groups and the maximum size by the combination of the largest pebbles. So, the coagulated mass will be distributed into size bins ranging from $\left(a_{p, i, j \min }^{3}+a_{p, i, k \min }^{3}\right)^{1 / 3}$ to $\left(a_{p, i, j \max }^{3}+a_{p, i, k \max }^{3}\right)^{1 / 3}$.

The coagulation time step also follows a substep algorithm in each mesh, with the condition that $\Delta t$ is chosen so that the fraction of pebbles (by number or mass) that coagulate is no larger than 0.02 .

For our simulation domain, we only consider pebble evolution in the region outside of the pressure maximum of the DZIB, i.e., from about 0.1-30 au.

The outer boundary of $30 \mathrm{au}$ is chosen as a representative outer disk scale, which contains a large enough dust reservoir to form a system of planets for the various disk models considered. However, this choice is somewhat arbitrary and, as we will mainly focus on the results of models that involve a steady injection of small pebbles at this outer boundary, the initial reservoir of solid material is not particularly important.

The radial spatial resolutions of the model is set by 160 logarithmically divided grids, from 0.1 to $30 \mathrm{au}$. We follow pebbles from a minimum particle radius of $a_{p \text {, min }}$, while solids below this limit are considered to be "dust." While $a_{p, \min }=$ $0.01 \mathrm{~cm}$ is the fiducial choice, we also consider models with $a_{p, \min }=0.1 \mathrm{~cm}$ to explore the dependence of our results on this choice. Note that we use logarithmically spaced grids in pebble-size space: $a_{p, j}=a_{p, \min } \times 10^{j \times 0.05}$. At the start of the simulation, the dust mass surface density $\Sigma_{d}$ is set to be $1 \%$ of the gas mass surface density, $\Sigma_{g}$. The initial pebbles are given a mass surface density of $\Sigma_{p}$ that is $1 \%$ of $\Sigma_{d}$ (in the fiducial case; variations of this parameter are also explored). The distribution of the radii of the initial/injected pebbles is set to range from $a_{p, \text { min }}$ to $0.3 \mathrm{~cm}$ with a number density versus size distribution following the equilibrium distribution of Birnstiel et al. (2015),

$$
d n_{p}\left(a_{p}\right) \propto a_{p}^{-5 / 8} d a_{p}
$$

At the outer boundary, the disk is supplied with dust and pebbles given the steady-state accretion rate and our adopted value of $f_{s}=0.01$, and the fiducial pebble to dust mass ratio is again $1 \%$ for the injected solids.

\subsection{Fiducial Model Results and Effects of Accretion Rate, Initial Pebble Distribution, and Minimum Pebble Size}

We first present the results in Figure 7 for the time evolution of the mass flux of pebbles delivered to the inner disk inner boundary $(r=0.1 \mathrm{au})$ for disks with viscosity parameter $\alpha=10^{-4}$. Our fiducial case adopts a minimum pebble radius of $a_{p, \text { min }}=0.01 \mathrm{~cm}$ (left-column panels of Figure 7), but we also show the results for $a_{p, \text { min }}=0.1 \mathrm{~cm}$ (right column). Our fiducial choice of initial and injected pebble to dust mass surface densities is $\Sigma_{p} / \Sigma_{d}=0.01$ (middle row), but we also show the results for ratios that are three times smaller (top row) and larger (bottom row). Our fiducial case has a steady accretion rate of $\dot{m}=10^{-9} M_{\odot} \mathrm{yr}^{-1}$ (black/gray lines), but we explore the results of varying this from $10^{-10} M_{\odot} \mathrm{yr}^{-1}$ (red lines) to $10^{-8} M_{\odot} \mathrm{yr}^{-1}$ (blue lines). We show the results of the case of pebble evolution where only the sweep-up growth of dust is considered (solid lines), and then also for the case where pebble-pebble coagulation is included (dashed lines). Finally, our fiducial model involves the initial disk from 0.1 to 30 au being populated with pebbles with the given ratio of $\Sigma_{p} / \Sigma_{d}$ (dark lines); however, we also consider the case of an 
(a)

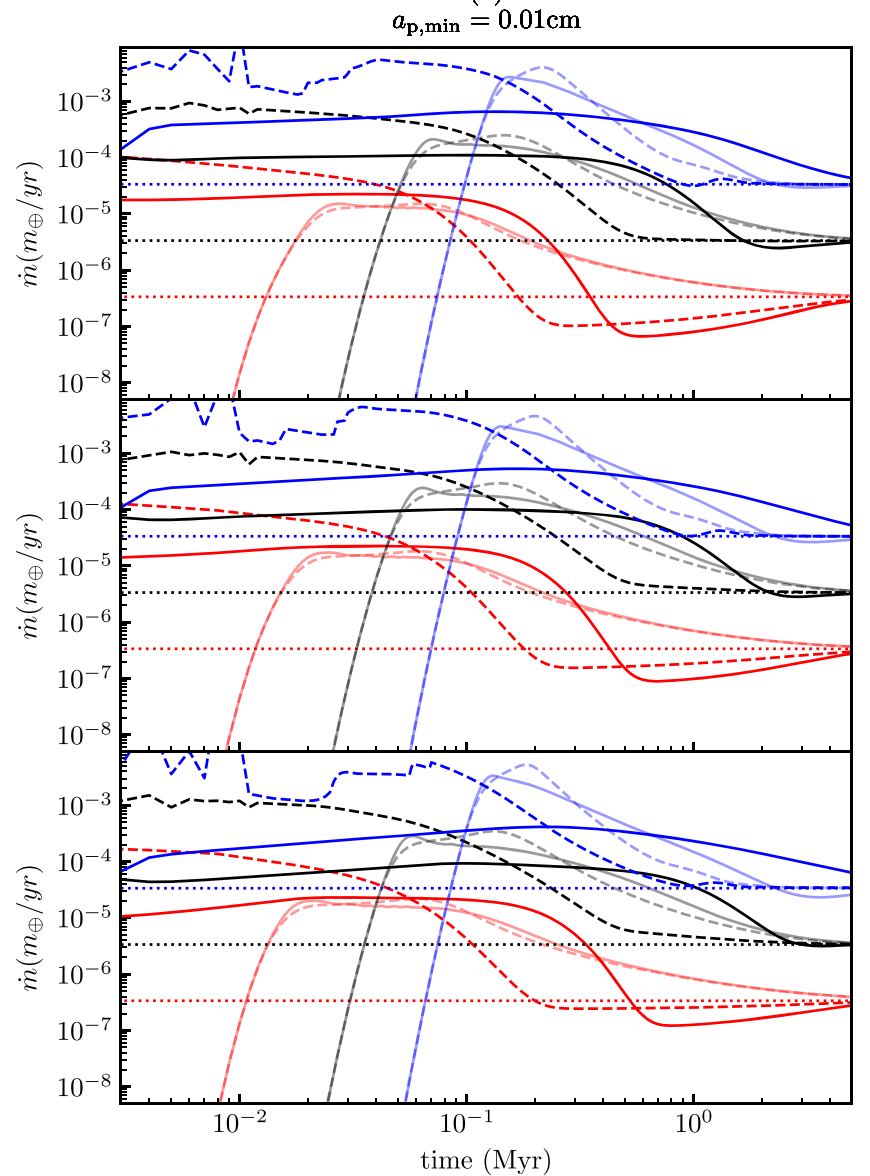

(b)

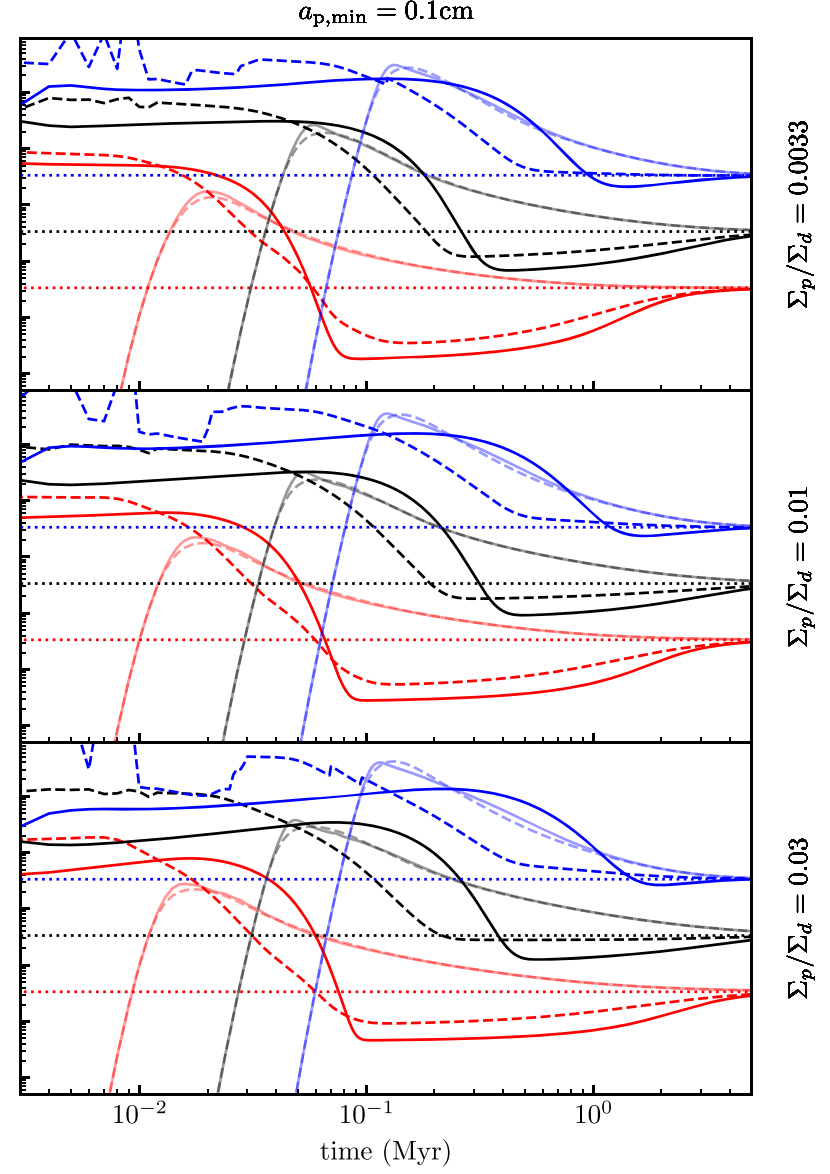

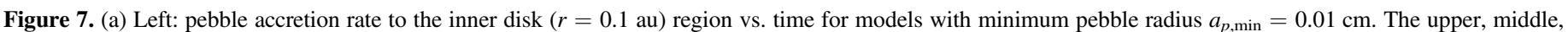

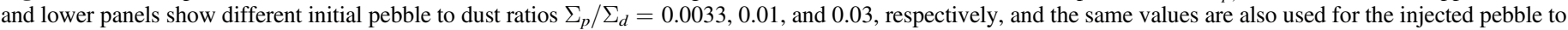

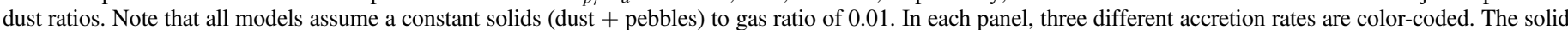

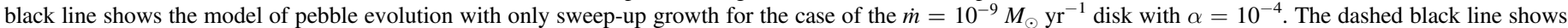

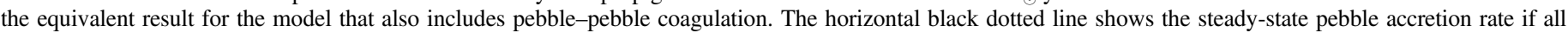

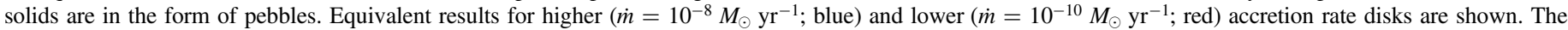

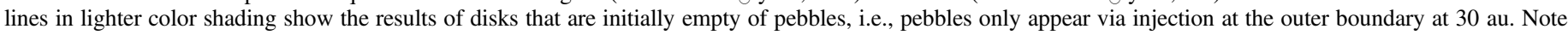

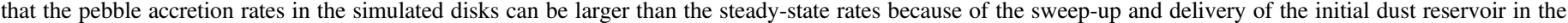
disk, but the asymptotic behavior at late times is toward this steady-state rate. (b) Right: same as panel (a) but now for $a_{p, \text { min }}=0.1 \mathrm{~cm}$.

initially "empty" disk, i.e., no pebbles, only dust, with pebbles only appearing via injection at 30 au (lighter shaded lines). The steadystate pebble supply rates assuming all solids are in pebbles are shown by the horizontal dotted lines in the panels, i.e., three lines for the three accretion rates of $10^{-10}, 10^{-9}$, and $10^{-8} M_{\odot} \mathrm{yr}^{-1}$.

Considering the fiducial case in the left-middle panel, we see that there is an initial period of pebble supply rates that is greater than the steady-state rate due to the delivery of solid material (mostly dust) that was initialized to be present in the disk, i.e., from 0.1 to 30 au. Pebble-pebble coagulation leads to more efficient sweep-up of this material so that the period of elevated supply rate is shorter but more intense. The late-time behavior of the models is an asymptotic approach to the steadystate supply rate (from above).

This trend is basically repeated in the higher- $\dot{m}$ case. However, in the lower- $\dot{m}$ case, we see that the pebble supply rate drops below the expected steady-state rate, and then slowly approaches it from below. Figure 7(b) shows the same models, but now for $a_{p, \min }=0.1 \mathrm{~cm}$, which has the effect of initializing and continuing the injection of a smaller number of larger pebbles. A similar behavior is seen, with an initial "spike" phase of elevated pebble supply rates, which are shorter, but more intense, if pebble-pebble coagulation is modeled. Now, however, the $\dot{m}=10^{-9} M_{\odot} \mathrm{yr}^{-1}$ case also exhibits a pebble supply rate that falls below the steady-state supply rate immediately after the spike phase.

The above behavior can be better understood by comparing it to the same results in lighter colored lines that show the models in which there are no initial pebbles in the disk: they only appear via injection at the 30 au boundary. These models show the initial elevated pebble supply rates due to the delivery of the initial dust from the disk, but they do not show the decremented phases where the pebble accretion rate is below the steady-state rate.

We conclude that the initial spike phase, due to the delivery of initial disk dust, is a somewhat artificial feature of our model, dependent on the initial conditions. A subsequent decremented supply rate phase can occur in some cases if there has been a very efficient sweep-up of the initial dust by an initial population of pebbles and there is a relatively long period of time needed for the injected pebbles to drift in to the inner disk. The limiting case of no initial pebbles in the disk is instructive in that it tells us the time needed for the disk to reach a quasi-equilibrium state where the pebble supply rate at 

$10^{-4}$
$10^{-3}$
$10^{-2}$
$10^{-1}$
$10^{0}$
$10^{1}$
$10^{2}$

$\Sigma / \operatorname{dlog} a_{p}\left(\mathrm{gcm}^{-2} \mathrm{dex}^{-1}\right)$

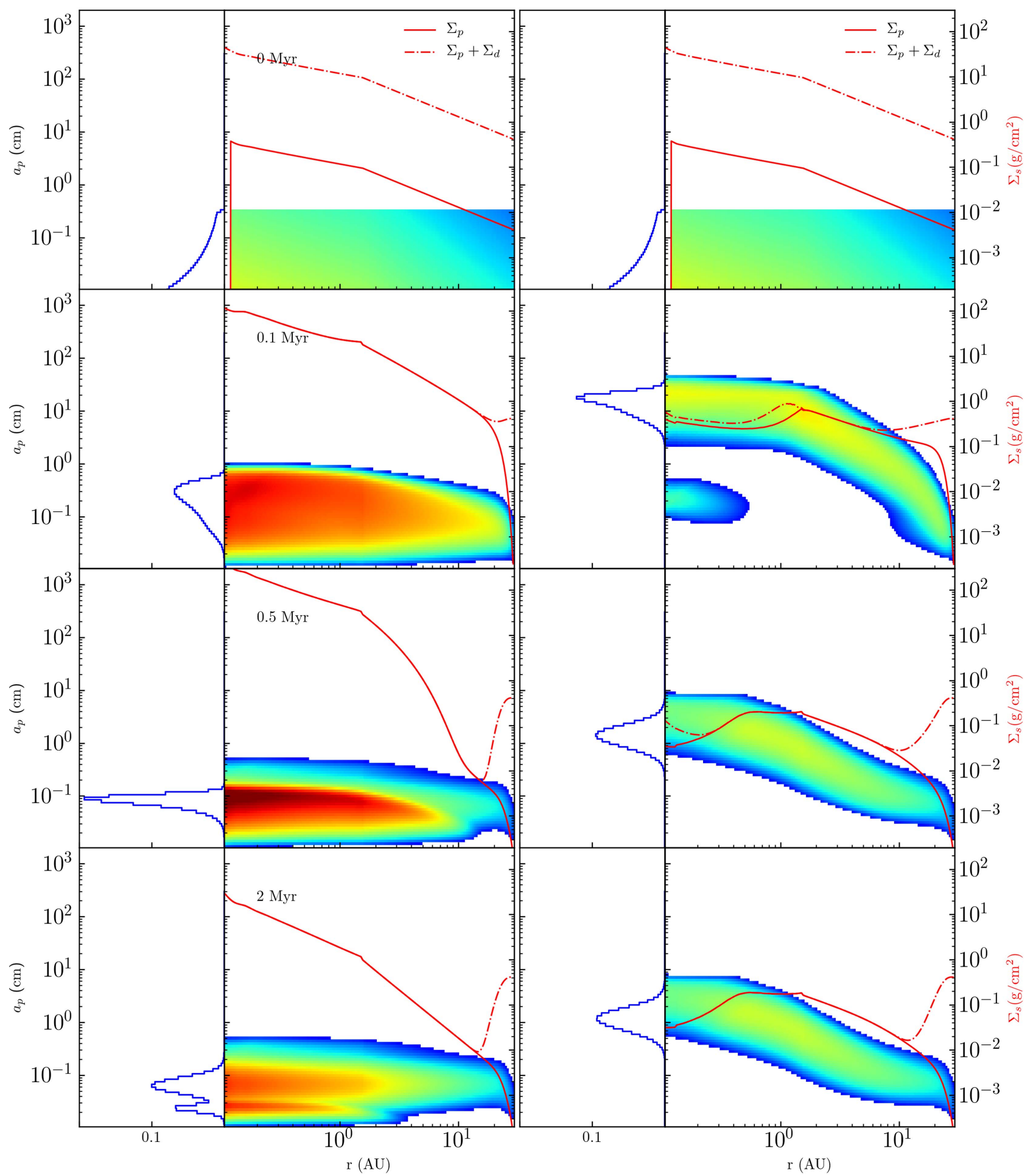

Figure 8. Evolution of pebble size vs. orbital radius distributions from the initial condition (top row), $0.1 \mathrm{Myr}$ (second row), $0.5 \mathrm{Myr}$ (third row), to $2 \mathrm{Myr}$ (bottom row). The left column shows the sweep-up-only growth model. The right column shows the sweep-up plus pebble-pebble coagulation growth model. In each panel, the color scale shows the distribution of the pebbles, while on the left there is a projected histogram of the sizes of those pebble inside 1 au radii. The red solid line shows the mass surface density of pebbles. The red dotted-dashed line shows the total mass surface density of solids (dust plus pebbles). When these overlap, most of the solid mass is in pebbles, which is achieved by late times in both models.

the inner boundary equals that injected at 30 au. For only sweep-up growth, this timescale can be $\gtrsim 1$ Myr for the high accretion rate case, and somewhat shorter for lower- $\dot{m}$ cases.
However, including pebble-pebble coagulation reduces these timescales. Since early high accretion rate phases are expected to last $\lesssim 1 \mathrm{Myr}$, i.e., the evolution from the protostellar disk 
(a)

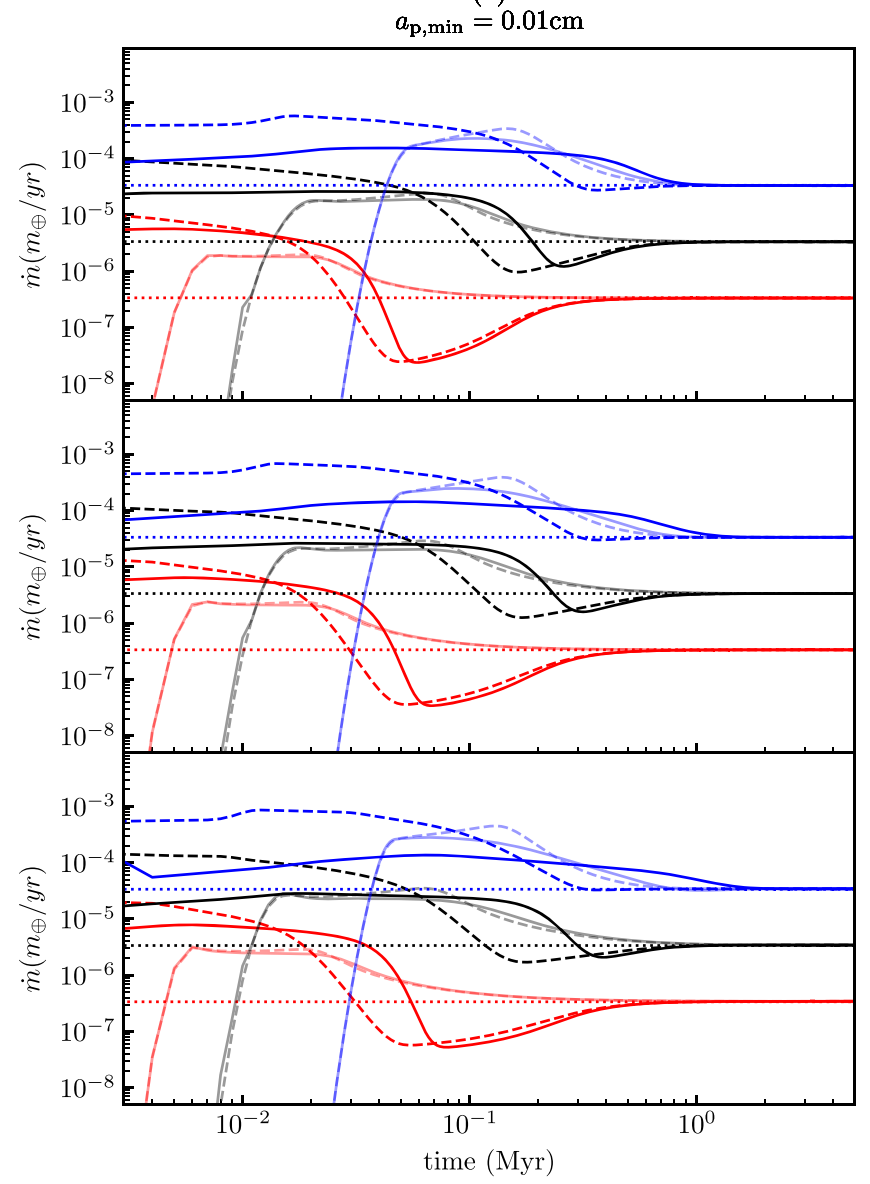

(b)

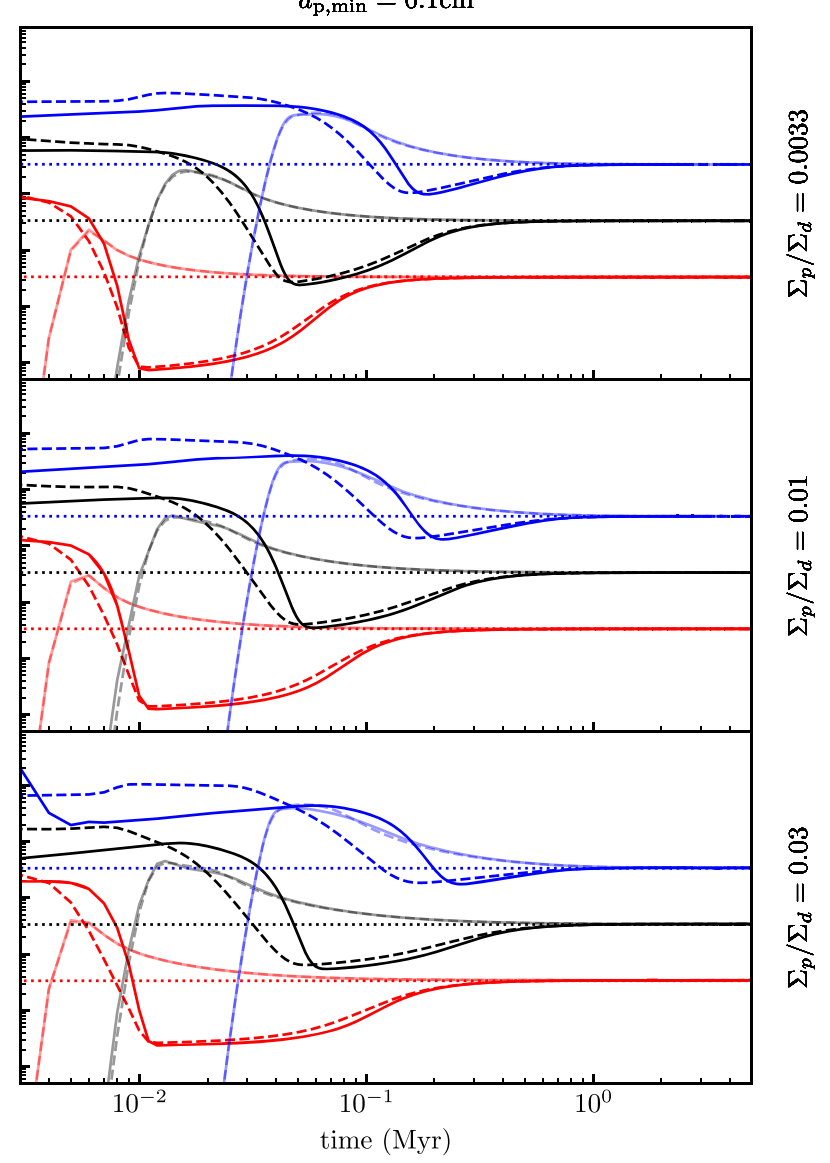

Figure 9. (a) Left: same as Figure 7(a), but now for disks with $\alpha=10^{-3}$. (b) Right: same as panel (a) but now for $a_{p \text {,min }}=0.1 \mathrm{~cm}$.

phase, this tells us that a globally self-consistent model may need to take into account the evolving disk structure due to a declining accretion rate and that a steady state may not be reached in certain circumstances.

Figure 8 shows the radial profiles of the dust-size distributions for our fiducial model with and without pebblepebble coagulation. The size distribution of pebbles delivered to the inner disk is significantly larger in the latter case. The figure also shows the radial profiles of the mass surface densities of pebbles and total solids, which allows the mass fraction of solids that is in pebbles to be assessed compared to dust (recall that our modeling is of the midplane region).

In models with pebble coagulation, the sweeping up of dust is not as efficient in the inner disk as in the sweep-up-only model. As seen from Figure 8, the pebble mass surface density is significantly lower than the total mass surface density of solids, while in the sweep-up-only model, pebbles dominate the total solid mass starting even at $0.1 \mathrm{Myr}$. The growth rate of pebble coagulation scales with the square of the pebble number density $N_{p}^{2}$, while sweep-up growth scales with $N_{p}$. During the early stages in the inner disk, where the pebble density is high, fast mutual coagulation produces large, Stokes-limited pebbles without sweeping up much dust. The inner disk can maintain a high dust fraction until the pebble flux from the outer disk arrives.

Another feature worth noticing in the coagulation model is the transient behavior at $0.1 \mathrm{Myr}$. The pebbles are divided into two groups in the inner disk. There are two contributing factors: the Stokes-limited sweep-up growth forbids the further sweep-up growth of some initial pebbles in the inner disk, and the $15 \mathrm{~m} \mathrm{~s}^{-1}$ coagulation speed limit stops them from being coagulated into larger sizes, while their mutual coagulations are very inefficient due to small cross-sections and low relative velocities (see Figure 6).

\subsection{Effects of a Viscosity}

The appropriate value of the effective viscosity, parameterized via $\alpha$, is very uncertain. Here we repeat the analysis of the previous subsection, but now for disks with $\alpha=10^{-3}$. These results are shown in Figure 9. In our modeling, the variation of $\alpha$ plays a similar role to $\dot{m}$ as it mainly affects mass surface density, i.e., $\Sigma_{g} \propto \alpha^{-4 / 5}$ (see Equation (9)). Higher- $\alpha$ disks thus have a smaller initial dust reservoir, i.e., on the scale of the 30 au disk, and less efficient sweep-up growth. Thus, note that the pebble flux profile in a disk of $\dot{m}=10^{-9} M_{\odot} \mathrm{yr}^{-1}, \alpha=10^{-3}$ is quite similar to the one with $\dot{m}=10^{-10} M_{\odot} \mathrm{yr}^{-1}, \alpha=10^{-4}$. Although the dust in the inner disk transfers mostly into pebbles, as in the low- $\alpha$ case, the solids in the outer disk stay mostly in the form of dust for a much longer time. This effect is visible in Figure 10, which shows the radial dependence of the pebble-size distributions and the relative levels of $\Sigma_{p}$ and $\left(\Sigma_{p}+\Sigma_{d}\right)$ for the higher- $\alpha$ disks. 

$10^{-4}$
$10^{-3}$

$10^{-2}$

$10^{-1}$

$10^{0}$

$10^{1}$

$10^{2}$

$\Sigma / \operatorname{dlog} a_{p}\left(\mathrm{gcm}^{-2} \mathrm{dex}^{-1}\right)$

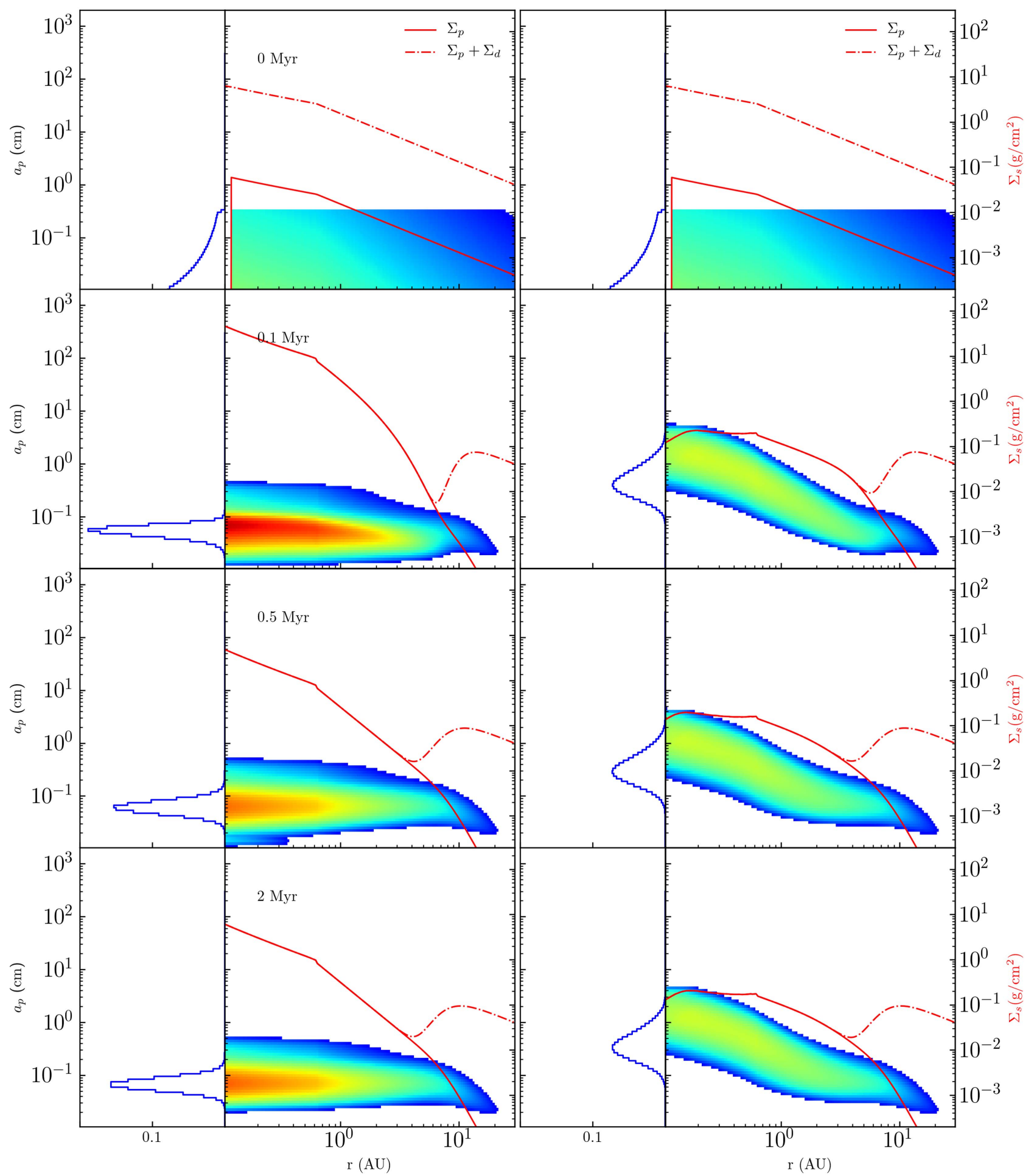

Figure 10. Same as Figure 8, but showing disks with viscosity parameter $\alpha=10^{-3}$. The major difference is in the outer disk: significantly more solids remain in the form of dust than in the disk with $\alpha=10^{-4}$, thus resulting in a lower pebble flux after the dust in the inner disk has been swept up.

\section{Assembling an STIP}

\subsection{Steady Accretion Rate Disks}

We now consider the implications of these models for the timescale of assembling an entire STIP. As a specific example, we will consider the ability of disk models to form a five-planet system and the time it takes them to do so. We compare the accumulated mass from the delivered inner disk pebble flux to the required mass of the planets, i.e., that set by the gapopening condition for $M_{\mathrm{G}, \mathrm{D}}$ from Equation (15). 
A noticeable feature of our modeled pebble flux is the "spike" phase at the beginning of the simulations, which can transport a large amount of solids $\left(\sim 50 M_{\oplus}\right)$ in less than 0.5 Myr. However, this spike feature mostly reflects the adopted initial condition of pebbles and dust in the inner $(\lesssim 10 \mathrm{au})$ disk. Since these solids in reality could have already drifted in at an earlier phase of the disk's evolution, here we ignore the pebbles delivered in the early spike phase up to 0.1 Myr. Still, we will see that our results do depend on how the initial conditions of the disks are set up.

In the context of IOPF, building an STIP starts with the innermost "Vulcan" planet at $r_{1200 \mathrm{~K}}$. After that, here, for simplicity, we assume that the orbit of each subsequent planet is 20 Hill radii away from the previously formed planet, which is close to the median value from observations (Paper I) and within the range of the DZIB retreat distances modeled in Paper III as being due to increased ionization via the penetration of protostellar X-rays. The planet mass is calculated as $M_{\mathrm{G}, \mathrm{D}, 1.44}$ using Equation (15). We simply assume that all accumulated mass of pebbles will be transferred into planets, i.e., the radially drifting pebbles will be caught by the DZIB pressure trap and later accreted with $100 \%$ efficiency by the protoplanet.

The results of this modeling for our fiducial disk $\left(\dot{m}=10^{-9} M_{\odot} \mathrm{yr}^{-1}, \alpha=1 \times 10^{-4}\right)$ are shown with solid lines in the left pair of panels in the middle row of Figure 11. Here, the first planet starts forming $0.1 \mathrm{Myr}$ after the spike phase, and the fifth has completed formation within the following $0.4 \mathrm{Myr}$ in the case of sweep-up-only pebble evolution, and within $1.5 \mathrm{Myr}$ in the case including pebblepebble coagulation (blue dots mark the time of formation of each planet). The difference is due to the different pebble supply rates, with the first model more heavily influenced by the initial spike phase from the initial dust reservoir. The orbital distribution and masses of the planets are shown in the rightmost panel of the middle row (again shown as blue dots).

Equivalent models with $\alpha=1 \times 10^{-3}$ tend to have lower pebble supply rates (dashed lines). If this value of $\alpha$ also holds in the DZIB region, then the gap-opening mass is increased, implying more massive planets. In this case, only two such planets (green dots) have time to form within 5 Myr.

The top and bottom rows of Figure 11 show equivalent results for $\dot{m}=10^{-8} M_{\odot} \mathrm{yr}^{-1}$ and $\dot{m}=10^{-10} M_{\odot} \mathrm{yr}^{-1}$, respectively. Higher/lower accretion rates imply higher/lower gapopening masses, but with a sublinear scaling. The total mass of pebbles delivered to the inner region in a given time scales more steeply with accretion rate. Thus, it becomes more difficult for lower accretion rate disk models to be able to form the entire five-planet system. Overall, when comparing models with different $\dot{m}$ and $\alpha$, we find that to form a realistic STIP, the disk should have $\dot{m} \sim 10^{-9} M_{\odot} \mathrm{yr}^{-1}$ and a viscous $\alpha$ no more than $\sim 2 \times 10^{-4}$.

\subsection{Evolving Disks}

The lifetimes of protoplanetary disks are estimated to range from $\sim 1$ to 10 Myr (e.g., Alexander et al. 2014; Espaillat et al. 2014). The effects of an evolving disk have been previously considered in dust evolution models. For example, Birnstiel et al. (2010) set up a disk model subject to viscous evolution and gravitational instability, starting from the very early infall phase. It gives a relatively constant accretion rate starting from 0.15 to $1 \mathrm{Myr}$. Other methods involve adopting simple functional forms for the evolution of the accretion rate, such as power-law or exponential decay (e.g., Hueso \& Guillot 2005; Bitsch et al. 2015). The disk modeled by Bitsch et al. (2015) has an accretion rate that evolves as

$$
\dot{m}=\dot{m}_{0} e^{-t / t_{0}},
$$

with $\dot{m}_{0}=7.24 \times 10^{-9} M_{\odot} \mathrm{yr}^{-1}$ and $t_{0}=3.1 \times 10^{5} \mathrm{yr}$. We adopt the same functional form, but with $\dot{m}_{0}=1.0 \times$ $10^{-9} M_{\odot} \mathrm{yr}^{-1}$ and $t_{0}=4.34 \times 10^{5} \mathrm{yr}$, so that the accretion rate declines by a factor of 10 in $1 \mathrm{Myr}$.

In our numerical modeling, we update the disk properties, i.e., the accretion rate, every 1000 years. Note that in such models of exponentially declining accretion rate, the disk evolution is not fully self-consistent for its accretion history, i.e., the declining $\Sigma_{g}$ does not match the viscous radial drift of gas. Thus, these models should be viewed as being simple "toy" models of disk evolution. Note also that when we force the gas disk properties to evolve with an exponentially declining rate, we have a choice about how we treat the associated dust that may be mixed together with the gas. We consider two extreme cases. Case A: the dust content of the disk, i.e., $\Sigma_{d}$, does not evolve with the declining gas, i.e., it would remain at a steady mass surface density, except for the effects of radial drift (due to the assumed $\alpha$ viscosity) and sweep-up by pebbles. Case B, the dust content of the disk, i.e., $\Sigma_{d}$, is decreased with the same exponential decay rate of the gas, with this decay factor applied multiplicatively on top of any other changes due to radial drift and sweep-up by pebbles.

In principle, there are now several parameters that may be varied in these models: $\dot{m}_{0}, t_{0}, t_{\mathrm{IOPF}}$ (i.e., the time when the first planet starts to form), in addition to other model parameters already introduced, such as $\alpha$ and $a_{p, \text { min. }}$. Furthermore, there are the choices of either Case A or B, introduced above; whether or not pebble-pebble coagulation is included in the modeling; and whether or not the disk model is initially "empty" of pebbles. It is not our intention here to carry out a full exploration of the parameter space of all these models. Rather, here we present several example models of representative cases, in particular focusing on the examples of parameter combinations that produce realistic STIPs within reasonable timescales.

Following the format of Figure 11, Figure 12 now shows example models with $\dot{m}_{0}=10^{-9} M_{\odot} \mathrm{yr}^{-1}, t_{0}=4.34 \times 10^{5} \mathrm{yr}$, $t_{\mathrm{IOPF}}=0.1 \mathrm{Myr}$, and $a_{p, \min }=0.01 \mathrm{~cm}$. Case A is shown in the top row, and Case B in the bottom row. In each row, models with low $\alpha\left(10^{-4}\right)$, high $\alpha\left(10^{-3}\right)$, and "mixed" $\alpha$ (i.e., $10^{-4}$ in the DZIB region; $10^{-3}$ in the main disk region beyond the DZIB region that is relevant for pebble growth and supply) for the cases without and with pebble-pebble coagulation are shown. In these models, the value of $\alpha$ in the DZIB region has a direct effect on the mass of the planets needed to open up a gap, i.e., lower-mass planets in lower-viscosity disks. The value of $\alpha$ in the main disk region beyond the DZIB affects the global disk structure in the zone responsible for pebble growth and supply: in particular, lower viscosity implies higher disk mass for a given accretion rate, and thus a more massive reservoir of dust for planet formation. The "mixed" $\alpha$ model that is presented is a simple means of allowing different values of viscosity in the DZIB, which affect planet masses, and in the main disk region, which affect pebble growth and supply.

Compared to the steady-state disk models with $\dot{m}=$ $10^{-9} M_{\odot} \mathrm{yr}^{-1}$, these evolving disk models have lower latetime pebble delivery rates. Planets forming at these later times 

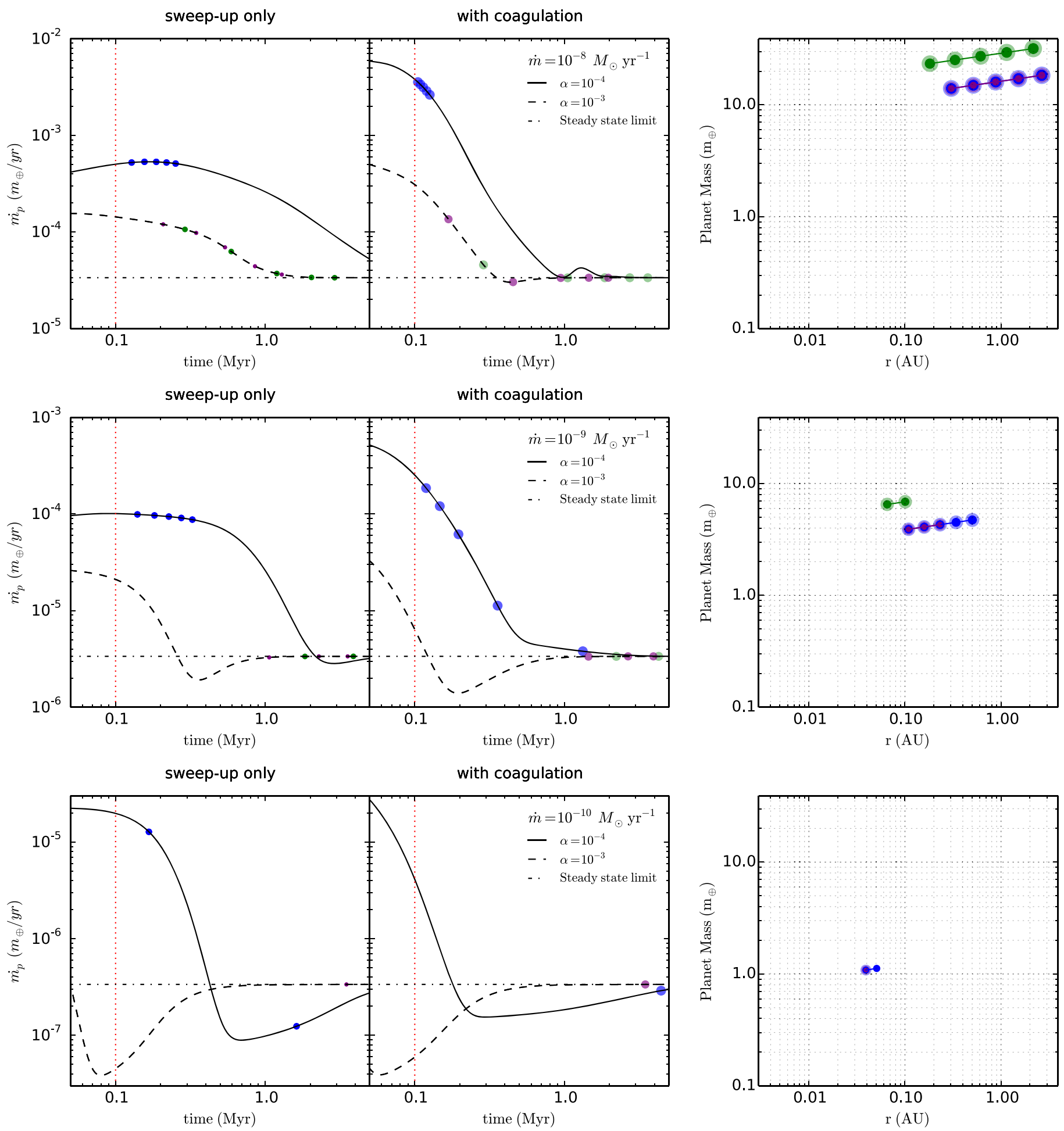

Figure 11. (a) Top row: example of STIP formation history (left and middle panels) and orbital architecture, i.e., planet mass vs. orbital radius (right panel), given a pebble supply rate history for a model with $a_{p, \min }=0.01 \mathrm{~cm}$ and a steady gas accretion rate of $\dot{m}=10^{-8} M_{\odot} \mathrm{yr}^{-1}$. The results of models with only sweep-up growth of pebbles are shown in the left panel. The black solid line shows the pebble supply rate to the inner disk with $\alpha=10^{-4}$, and the black dashed line shows the pebble supply rate with $\alpha=10^{-3}$. The dotted-dashed line marks the steady-state pebble flux limit. The colored dots on the pebble delivery lines indicate the formation time of each planet, with blue dots for $\alpha=10^{-4}$, green dots for $\alpha=10^{-3}$, and purple dots for global disk $\alpha=10^{-3}$ but with the calculation of $M_{\mathrm{G}, \mathrm{D}}$ done for $\alpha=10^{-4}$, i.e., a lower value of $\alpha$ near the DZIB. Note that in these models planet formation is assumed to start at $0.1 \mathrm{Myr}$ (marked by the vertical dashed line). The middle panel shows the equivalent results, but now including pebble-pebble coagulation. (b) Middle row: same as panel (a) but for disk accretion rates of $\dot{m}=10^{-9} M_{\odot} \mathrm{yr}^{-1}$. (c) Bottom row: same as panel (a) but for disk accretion rates of $\dot{m}=10^{-10} M_{\odot} \mathrm{yr}^{-1}$. The disks with lower accretion rates can fail to form all five planets within the 5 Myr period considered.

have lower masses, since the gap-opening mass is lower for lower accretion rates. We see that certain combinations of models lead to planet formation being spread out in time over a few Myr, which leads to a significant decline in planet mass with orbital radius. This is not a particular feature of observed STIPs, modulo uncertainties in inferring their masses from 

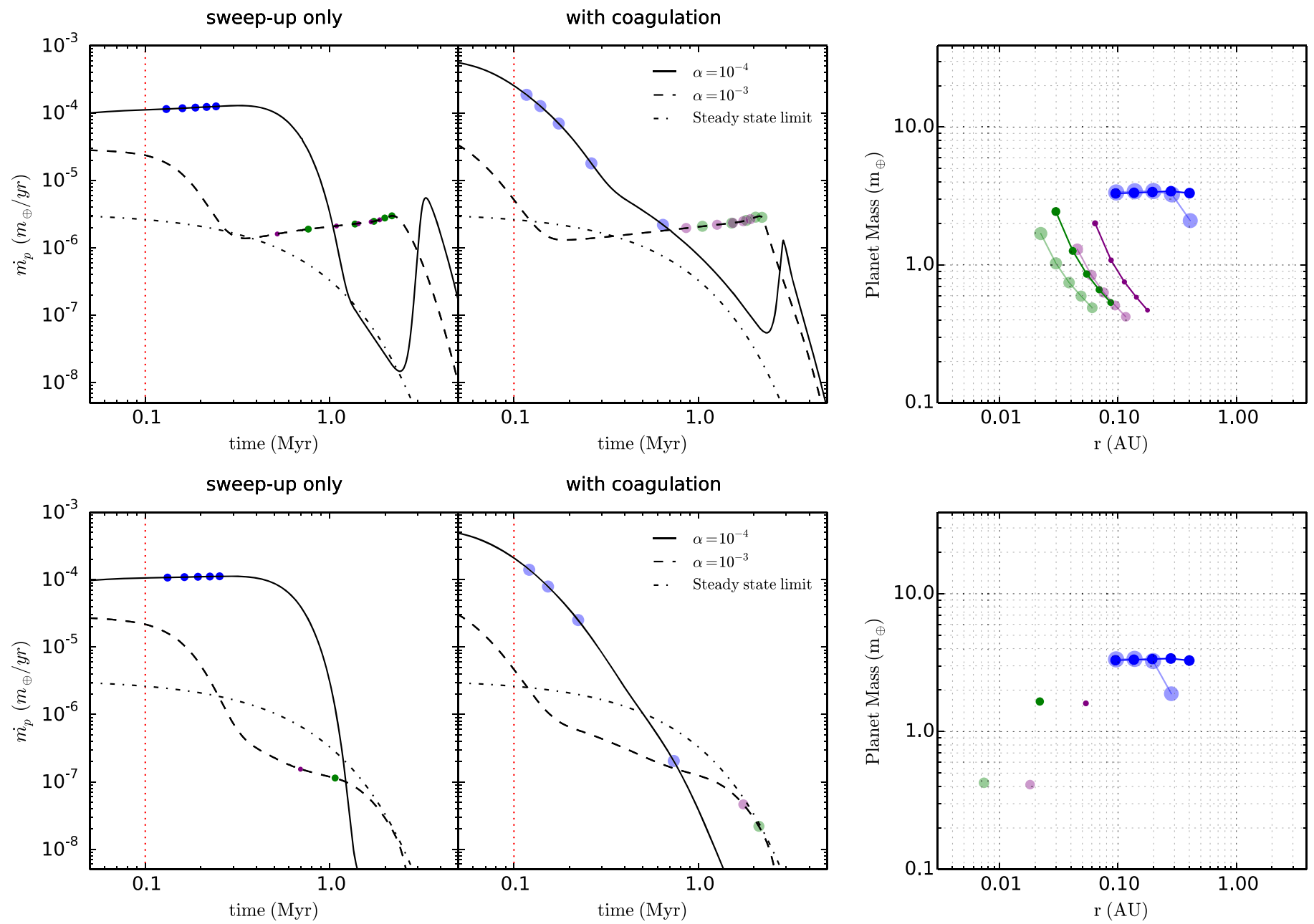

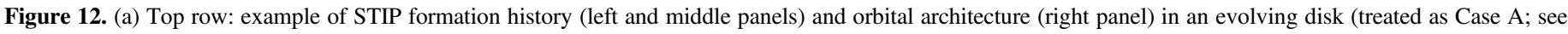

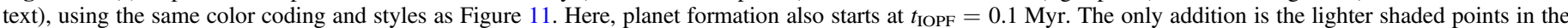

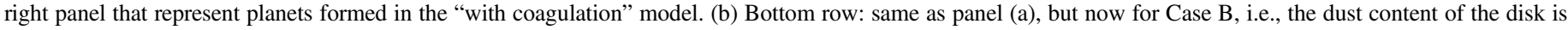
also scaled with the exponential decay function.

observed sizes (see, e.g., Paper I, II). To obtain relatively flat distributions of planet masses versus orbital radii requires IOPF to be mostly complete within $\sim t_{0}$ (and thus for $t_{\mathrm{IOPF}} \ll t_{0}$ ). Only the low- $\alpha$ models achieve these conditions.

The influence of the spike phase of the enhanced pebble supply rate at early times can extend the time period when reasonable STIPs can be formed by IOPF, especially in the sweep-up-only pebble evolution models. This is also illustrated in Figure 13, which shows the same results as in Figure 12, but now for $t_{\mathrm{IOPF}}=0.5 \mathrm{Myr}$. Only in the low- $\alpha$, sweep-up-only models does an STIP with a flat mass versus orbital radius distribution form.

We note that the flux increase after about $2.5 \mathrm{Myr}$ in Case A is a result of both leftover dust from early phase and the relatively large Stokes numbers of the injected pebbles at late stages. The injected pebbles, i.e., with radii of $0.01-0.3 \mathrm{~cm}$, have relatively small Stokes numbers in the early phases, when gas densities are higher. However, the Stokes number increases significantly as $\dot{m}$ drops. Still, in the models considered here, this late-time pulse of delivered pebbles is not significant compared to the early pebble delivery that formed the STIP.

Note that these are systems with modest initial accretion rates: $\dot{m}=10^{-9} M_{\odot} \mathrm{yr}^{-1}$. If IOPF starts at even higher $\dot{m}$, then the planets formed would be significantly more massive than typical observed STIPs (unless the DZIB $\alpha$ is much smaller than the values we have considered).

\section{Discussion and Conclusions}

In this paper, we have presented improved models of IOPF, with a particular focus on understanding if most solids will be delivered to the inner disk in the form of pebbles (i.e., the raw materials for planet formation) and then the implied formation times and constraints on disk properties during the time of IOPF. The models presented here are an attempt at calculating the "IOPF population synthesis" and can be compared to other similar models based on other theoretical frameworks (e.g., Ida $\&$ Lin 2004; Bitsch et al. 2015).

We first constructed improved disk models, extending to $30 \mathrm{au}$, especially using realistic opacities and including the effect of the active disk to passive disk transition. Next, we expanded on the work of Paper III to examine the gap-opening condition (i.e., the pressure maximum displacement from the planet) over a wider range of viscosities (especially extending to the low- $\alpha$ regime that seems to be needed for IOPF) and accretion rates. We find that Duffell's (2015) analytic description of the gap-opening criterion is a better description 

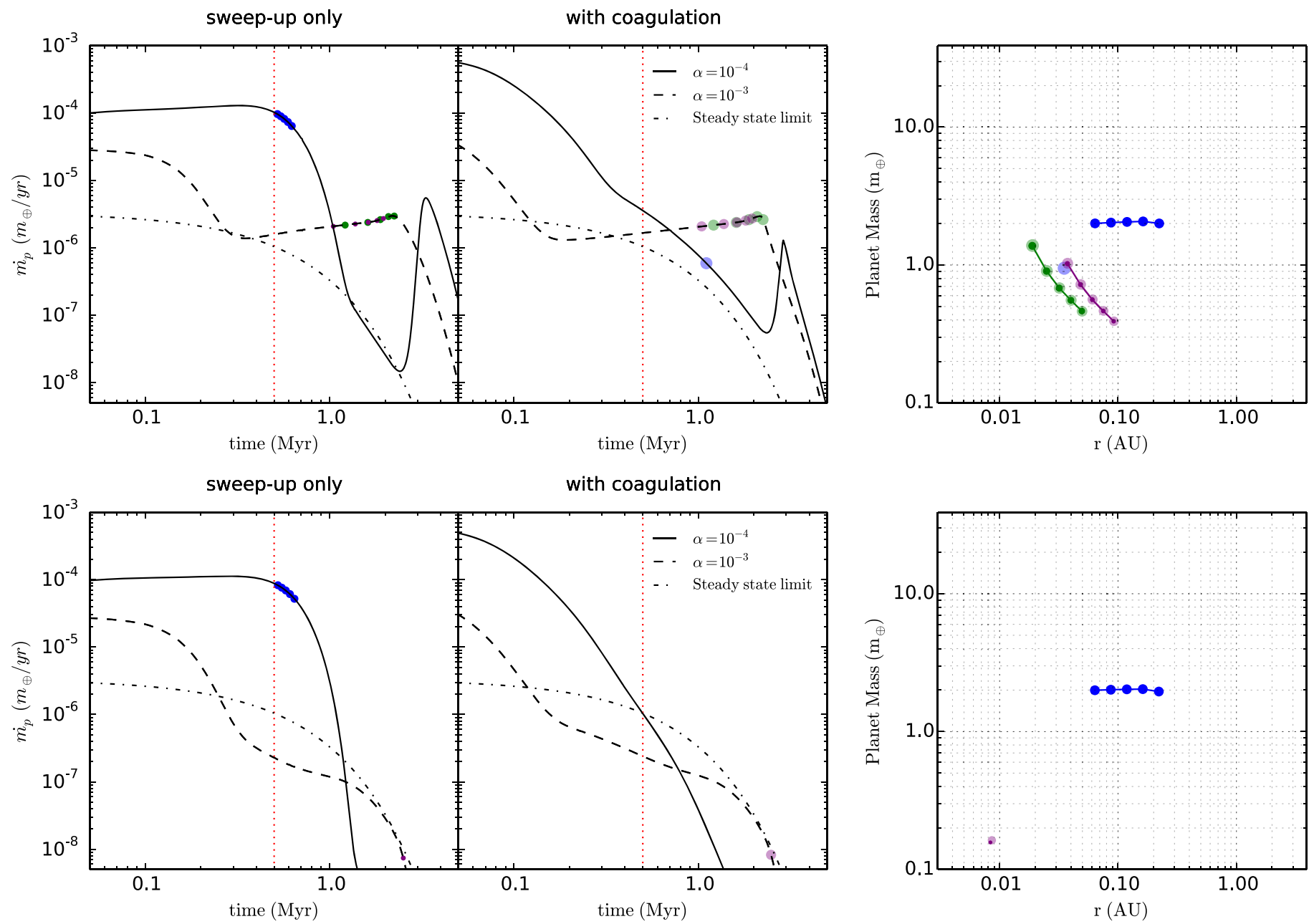

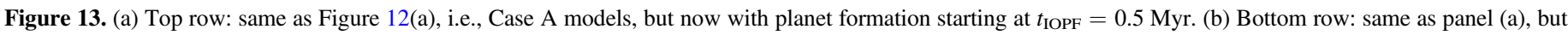
now for Case B, i.e., the dust content of the disk is also scaled with the exponential decay function.

of the results than the simple viscous criterion we used previously in Papers I to III. This implies that planets are more massive in the low- $\alpha$ regime, i.e., still $\sim 4 M_{\oplus}$ for $\alpha=10^{-4}$ for $\dot{m}=10^{-9} M_{\odot} \mathrm{yr}^{-1}$.

Next, we examined pebble evolution in these disks, presenting models for how a single pebble is expected to evolve via Stokes-limited sweep-up of dust and pebble-pebble coagulation. This formed the basis of a global pebble evolution model, i.e., of a population of pebbles, for given choices of initial conditions and boundary conditions (i.e., the injected pebbles at $30 \mathrm{au}$ ).

Simple constraints on the timescales of the first "Vulcan" planet formation were derived with the single pebble evolution model, under the assumption of a given efficiency of solids being incorporated into pebbles (Table 1). Fiducial estimates based on drift times from the reservoir radius are $\sim 10^{5} \mathrm{yr}$ to form the first planet. The global pebble evolution models showed that large, near-unity fractions of solids are expected to be incorporated into pebbles (with the caveat that our disk models apply to the midplane layer conditions; but this is where most solids are expected to have settled). By the time they reach the inner astronomical unit, pebble sizes are typically $\sim 0.1 \mathrm{~cm}$ in the sweep-up-only models and about a few centimeters in the models including pebble-pebble coagulation. Such predictions are potentially testable by observations of protoplanetary disks (e.g., Pérez et al. 2012; Trotta et al. 2013; Testi et al. 2014; Tazzari et al. 2016).

We utilized the global pebble evolution models in disks with steady accretion rates to predict pebble delivery rates to the inner disk. We showed the effects of model assumptions, including whether the disk is initially populated with pebbles. The models show an initial "spike" phase of an elevated pebble flux compared to the steady-state rate due to the sweeping up of the initial dust reservoir, which decays away in about a Myr.

Finally, we used these models to predict the formation history of STIPs forming via IOPF. The formation locations of the Vulcan planets were set self-consistently at $r_{1200 \mathrm{~K}}$ and with masses that depend on accretion rate, location, and disk viscosity. The timescale to form a five-planet system was assessed, with an overall cap of $5 \mathrm{Myr}$ imposed. In steady accretion rate disks, these models showed a preference for $\dot{m} \sim 10^{-9} M_{\odot} \mathrm{yr}^{-1}$. Higher accretion rate models form planets that are too massive and too far away from the star. Lower accretion rate models do not have pebble delivery rates high enough to form several planets. Extending to evolving disk models introduces additional parameters and model choices. For simple evolving disk models treated with an exponentially declining accretion rate, we require IOPF to be completed within this decay time, $\sim 1 \mathrm{Myr}$, in order to preserve the observed relatively flat scaling of planet mass with orbital radius. 
We note that IOPF's apparent preference for accretion rates of $\dot{m} \sim 10^{-9} M_{\odot} \mathrm{yr}^{-1}$ is quite consistent with the observed accretion rates of young stellar objects, including transition disk systems (e.g., Williams \& Cieza 2011; Manara et al. 2014, 2016). At even lower accretion rates, the rate of photoevaporation of the disk may become comparable, potentially terminating or greatly reducing the supply of gas to the inner disk (e.g., Ercolano et al. 2009; Owen et al. 2012; Ercolano et al. 2014). However, if the solids mostly settle on the disk midplane in the form of pebbles, as indicated by the results of our global pebble evolution models, then their supply is less likely to be affected by photoevaporation. Nevertheless, a more complete global evolution model of IOPF, planned for a future study, should also account for additional processes such as photoevaporation to examine its effects on gas supply to the inner disk region. Self-consistent constraints of disk lifetimes that are predicted by either residual core infall and/or photoevaporation will also need to be considered in this modeling.

We thank an anonymous referee for helpful comments that improved the clarity of this paper. J.C.T. acknowledges NASA ATP grant NNX15AK20G and NSF AAG grant 1616300. Z.Z. acknowledges NASA ATP grant NNX17AK40G and a Sloan Research Fellowship. T.B. acknowledges funding from the European Research Council (ERC) under the European Unions Horizon 2020 research and innovation programme under grant agreement No. 714769. A.N.Y. acknowledges NSF AAG grant 1616929. S.M. and J.C.T. acknowledge a Royal Society International Exchange grant.

\section{ORCID iDs}

Xiao Hu (胡晓) (10 https://orcid.org/0000-0003-3201-4549 Jonathan C. Tan (1) https://orcid.org/0000-0002-3389-9142 Zhaohuan Zhu (朱照寰) (10 https://orcid.org/0000-00033616-6822

Sourav Chatterjee (i) https://orcid.org/0000-0002-3680-2684 Tilman Birnstiel (ํ) https://orcid.org/0000-0002-1899-8783 Subhanjoy Mohanty (1) https://orcid.org/0000-00029896-8313

\section{References}

Alexander, R., Pascucci, I., Andrews, S., Armitage, P., \& Cieza, L. 2014, in Protostars and Planets VI, ed. H. Beuther et al. (Tucson, AZ: Univ. Arizona Press), 475

Armitage, P. J. 2007, arXiv:astro-ph/0701485

Baruteau, C., Crida, A., Paardekooper, S. J., et al. 2014, in Protostars and Planets VI, ed. H. Beuther et al. (Tucson: Univ. Arizona Press), 667

Birnstiel, T., Andrews, S. M., Pinilla, P., \& Kama, M. 2015, ApJL, 813, L14 Birnstiel, T., Dullemond, C. P., \& Brauer, F. 2010, A\&A, 513, A79 Birnstiel, T., Klahr, H., \& Ercolano, B. 2012, A\&A, 539, A148 Bitsch, B., Lambrechts, M., \& Johansen, A. 2015, A\&A, 582A, 112B Bitsch, B., Morbidelli, A., Johansen, A., et al. 2018, arXiv:1801.02341 Ribas, Á, Bouy, H., \& Merín, B. 2015, A\&A, 576, A52 Brauer, F., Dullemond, C. P., \& Henning, T. 2008, A\&A, 480, 859 Chatterjee, S., \& Ford, E. B. 2015, ApJ, 803, 33C Chatterjee, S., \& Tan, J. C. 2014, ApJ, 780, 53 Chatterjee, S., \& Tan, J. C. 2015, ApJL, 798, L32
Chiang, E., \& Laughlin, G. 2013, MNRAS, 431, 3444

Chiang, E. I., \& Goldreich, P. 1997, ApJ, 490, 368

Cossou, C., Raymond, S. N., \& Pierens, A. 2013, A\&A, 553L, 2C

Cossou, C., Raymond, S. N., \& Pierens, A. 2014, in IAU Symp. 299 Exploring the Formation and Evolution of Planetary Systems, ed. M. Booth, B. C. Matthews, \& J. R. Graham (Victoria: Cambridge Univ. Press), 360

Duffell, P. C. 2015, ApJL, 807, L11

Duffell, P. C., \& MacFadyen, A. I. 2013, ApJ, 769, 41

Dullemond, C. P., \& Dominik, C. 2005, A\&A, 434, 971

Dumusque, X., Bonomo, A. S., Haywood, R. D., et al. 2014, ApJ, 789, 154

Dzyurkevich, N., Flock, M., Turner, N. J., Klahr, H., \& Henning, T. 2010, A\&A, 515, A70

Ercolano, B., Clarke, C. J., \& Drake, J. J. 2009, ApJ, 699, 1639

Ercolano, B., Mayr, D., Owen, J. E., Rosotti, G., \& Manara, C. F. 2014, MNRAS, 439, 256

Espaillat, C., Muzerolle, J., Najita, J., et al. 2014, in Protostars and Planets VI, ed. H. Beuther et al. (Tucson, AZ: Univ. Arizona Press), 497

Fabrycky, D. C., Lissauer, J. J., Ragozzine, D., et al. 2014, ApJ, 790, 146

Fang, J., \& Margot, J. L. 2012, ApJ, 761, 92

Fung, J., Shi, J.-M., \& Chiang, E. 2014, ApJ, 782, 88

Goldreich, P., \& Schlichting, H. E. 2014, AJ, 147, 32G

Hansen, B., \& Murray, N. 2012, ApJ, 751, 158

Hansen, B., \& Murray, N. 2013, ApJ, 775, 53

Hu, X., Tan, J. C., \& Chatterjee, S. 2014, in IAU Symp. 310, Complex Planetary Systems, ed. Z. Knězevíc \& A. Lemaitre (Namur: Cambridge Univ. Press), 66

Hu, X., Zhu, Z., Tan, J. C., \& Chatterjee, S. 2016, ApJ, 816, 19

Hueso, R., \& Guillot, T. 2005, A\&A, 442, 703

Ida, S., \& Lin, D. N. C. 2004, ApJ, 604, 388

Izidoro, A., Ogihara, M., Raymond, S. N., et al. 2017, MNRAS, 470, 1750

Kley, W., \& Nelson, R. P. 2012, ARA\&A, 50, 211

Krijt, S., Ormel, C. W., Dominik, C., \& Tielens, A. G. G. M. 2016, A\&A, 586, A20

Lambrechts, M., Johansen, A., \& Morbidelli, A. 2014, A\&A, 572A, 35L

Lin, D. N. C., \& Papaloizou, J. C. B. 1993, Protostars and Planets III (Tucson: Univ. Arizona Press), 749

Liu, B., Ormel, C. W., \& Lin, D. N. C. 2017, A\&A, 601, A15

Manara, C. F., Rosotti, G., Testi, L., Natta, A., et al. 2016, A\&A, 591, L3

Manara, C. F., Testi, L., Natta, A., et al. 2014, A\&A, 568, A18

Masset, F. S. 2000, A\&A, 141, 165

McNeil, D. S., \& Nelson, R. P. 2010, MNRAS, 401, 1691

Mohanty, S., Ercolano, B., \& Turner, N. J. 2013, ApJ, 764, 65

Mullally, F., Coughlin, J. L., Thompson, S. E., et al. 2015, ApJS, 217, 31M

Ogihara, M., Morbidelli, A., \& Guillot, T. 2015, A\&A, 578A, 36 O

Owen, J. E., Clarke, C. J., \& Ercolano, B. 2012, MNRAS, 422, 1880

Pérez, L. M., Carpenter, J. M., Chandler, C. J., et al. 2012, ApJL, 760, L17

Raymond, S. N., \& Cossou, C. 2014, MNRAS, 440L, 11R

Safronov, V. S. 1972, Evolution of the Protoplanetary Cloud and Formation of the Earth and Planets (Jerusalem: Keter Publishing House)

Sato, T., Okuzumi, S., \& Ida, S. 2015, arXiv:1512.02414

Schlichting, H. E. 2014, ApJ, 795L, 15S

Shakura, N. I., \& Sunyaev, R. A. 1973, A\&A, 24, 337

Tazzari, M., Testi, L., Ercolano, B., et al. 2016, A\&A, 588, A53

Testi, L., Birnstiel, T., Ricci, L., et al. 2014, in Protostars and Planets VI, ed. H. Beuther et al. (Tucson, AZ: Univ. Arizona Press), 339

Toomre, A. 1964, ApJ, 139, 1217T

Trotta, F., Testi, L., Natta, A., Isella, A., \& Ricci, L. 2013, A\&A, 558, A64 van der Marel, N., Verhaar, B. W., van Terwisga, S., et al. 2016, A\&A, 592, A126

Varnière, P., \& Tagger, M. 2006, A\&A, 446L, 13V

Williams, J. P., \& Cieza, L. A. 2011, ARA\&A, 49, 67

Windmark, F., Birnstiel, T., Güttler, C., et al. 2012, A\&A, 540, A73

Youdin, A. N., \& Goodman, J. 2005, ApJ, 620, 459Y

Zhu, Z., Hartmann, L., \& Gammie, C. 2009, ApJ, 694, 1045

Zhu, Z., Nelson, R. P., Dong, R., Espaillat, C., \& Hartmann, L. 2012, ApJ, 755,6

Zhu, Z., Stone, J. M., \& Rafikov, R. R. 2013, ApJ, 768, 143 